\title{
ANALYSIS OF BLOW-UP LOCUS AND EXISTENCE OF WEAK SOLUTIONS FOR NONLINEAR SUPERCRITICAL PROBLEMS
}

\author{
KELEI WANG AND JUNCHENG WEI
}

\begin{abstract}
We give a qualitative analysis of sequences of stationary solutions to the supercritical problem

$$
-\Delta u=|u|^{p-1} u \text { in } \Omega, \quad p>\frac{n+2}{n-2} .
$$

A consequence of the analysis is the existence of positive singular weak solutions on a convex domain when $p \in\left(\frac{n+2}{n-2}, \frac{n+1}{n-3}\right)$, with only isolated singularities.
\end{abstract}

\section{INTRODUCTION AND MAIN RESULTS}

Of concern is the local qualitative behavior of sequences of stationary weak solutions to

$$
-\Delta u=|u|^{p-1} u \text { in } B_{2}
$$

where $p>\frac{n+2}{n-2}$ and $B_{2}$ denotes the open ball in $\mathbb{R}^{n}$ with radius 2 . Throughout this paper $B_{r}(x)$ always denotes the open ball of radius $r$ with center at $x$, and $B_{r}$ is a ball centered at the origin.

For a weak solution $u \in H^{1}\left(B_{2}\right) \cap L^{p+1}\left(B_{2}\right)$, we say that it is stationary if for any smooth vector field $Y$ with compact support,

$$
\int\left(\frac{1}{2}|\nabla u|^{2}-\frac{1}{p+1}|u|^{p+1}\right) \operatorname{div} Y-D Y(\nabla u, \nabla u)=0 .
$$

For smooth solutions this condition follows from variations of the energy functional

$$
E(u)=\int \frac{1}{2}|\nabla u|^{2}-\frac{|u|^{p+1}}{p+1}
$$

with respect to perturbations of the parametrization of the domain, that is,

$$
\frac{d}{d t} E\left(\left.u(x+t Y(x))\right|_{t=0}=0\right.
$$

Key words and phrases. supercritical problems, blow-up locus, stationary solutions. 
Formula (1.2) can also be obtained by multiplying (1.1) by $Y \cdot \nabla u$ and integrating by parts, granted that this solution has enough regularity. Such condition is classical in many works dealing with partial regularity. In this problem it was first used by Pacard in [14, 15].

Since $p$ is assumed to be supercritical, solutions to (1.1) may not be smooth. Thus we need to define

Definition 1.1. For a solution $u$ of (1.1), its singular set $\mathcal{S}(u)$ consists of those points, such that in any neighborhood of this point $u$ is not bounded.

Roughly speaking $\mathcal{S}(u)=\{u=\infty\}$. By definition it is a closed set. Pacard's partial regularity result ([14]) says that for a stationary weak solution $u, \mathcal{S}(u)$ is a closed set satisfying

$$
H^{n-2 \frac{p+1}{p-1}}(\mathcal{S}(u))=0 .
$$

In particular, $\operatorname{dim}(\mathcal{S}(u)) \leq n-2 \frac{p+1}{p-1}$.

Let $u_{i} \in H^{1}\left(B_{2}\right) \cap L^{p+1}\left(B_{2}\right)$ be a sequence of stationary solutions to (1.1), with the energy bound

$$
\sup _{i} \int_{B_{2}}\left|\nabla u_{i}\right|^{2}+\left|u_{i}\right|^{1+p}:=M<+\infty .
$$

By this assumption, we can assume that $u_{i}$ converges weakly to $u$ in $H^{1}\left(B_{2}\right) \cap L^{p+1}\left(B_{2}\right)$. By the compact Sobolev embedding theorem, $u_{i}$ converges strongly to $u$ in $L^{q}\left(B_{2}\right)$ for any $q<p+1$. In particular $u_{i}^{p}$ converges to $u^{p}$ in $L^{1}\left(B_{2}\right)$, and $u$ is a weak solution to (1.1).

Denote the measure

$$
\mu_{i}=\left(\frac{p-1}{2}\left|\nabla u_{i}\right|^{2}+\frac{p-1}{p+1}\left|u_{i}\right|^{p+1}\right) d x .
$$

There exists a positive Radon measure $\nu$ such that,

$$
\mu_{i} \rightarrow\left(\frac{p-1}{2}|\nabla u|^{2}+\frac{p-1}{p+1}|u|^{p+1}\right) d x+\nu:=\mu \text { weakly as measures. }
$$

Note that $u_{i}$ converges strongly to $u$ in $H^{1}\left(B_{1}\right) \cap L^{p+1}\left(B_{1}\right)$ if and only if $\nu=0$ in $B_{1}$. Let

$$
\Sigma:=\mathcal{S}(u) \cup \operatorname{spt}(\nu),
$$

which we call the blow up locus of this sequence $\left(u_{i}\right)$.

The purpose of this paper is to give a qualitative characterization of the blow-up locus set. Denote $n-m$ to be the integer part of $n-2 \frac{p+1}{p-1}$. It is obvious that $m \geq 3$. With these notations we have 
Theorem 1.2. - For any $k, u_{i}$ converges to $u$ in $C_{l o c}^{k}\left(B_{1} \backslash \Sigma\right)$.

- If $n-2 \frac{p+1}{p-1}$ is not an integer, $\Sigma \cap B_{1}=\mathcal{S}(u)$ and $\nu=0$. Hence $u_{i} \rightarrow u$ strongly in $H^{1}\left(B_{1}\right) \cap L^{p+1}\left(B_{1}\right)$, and $u$ satisfies the stationary condition.

- If $n-2 \frac{p+1}{p-1}$ is an integer $n-m$, i.e., $p=\frac{m+2}{m-2}, \Sigma \cap B_{1}$ is $(n-m)$-countable rectifiable with $H^{n-m}\left(\Sigma \cap B_{1}\right)<+\infty$.

- If $n-2 \frac{p+1}{p-1}$ is an integer $n-m$ and $H^{n-m}\left(\Sigma \cap B_{1}\right)>0$, after extracting a subsequence, a rescaled subsequence of $u_{i}$ converges to a nontrivial smooth solution $\bar{v}$ to the low-dimensional Yamabe problem

$$
-\Delta \bar{v}=|\bar{v}|^{\frac{4}{m-2}} \bar{v} \quad \text { in } \mathbb{R}^{m}, \int_{\mathbb{R}^{m}}\left(|\nabla \bar{v}|^{2}+|\bar{v}|^{p+1}\right) \leq C(M) .
$$

Several remarks are in order: first, the above theorem implies that the only possible singularity formulation is through low-dimensional bubble (1.5). On the other hand, there are indeed sequences of bubbling solutions when $p$ is close to $\frac{m+2}{m-2}$ (del Pino-Musso-Pacard [4]). We conjecture that when $p=\frac{m+2}{m-2}$, the blow-up locus $\Sigma$ must be a minimal submanifold. (The problem is to show that the limit function $u$ is also stationary. This may not be true in general by examples of Ding-Li-Li [5] in harmonic map theory.) Second, Theorem 1.2 is reminiscent of similar results for harmonic maps by Lin [9]. Indeed our proof is motivated by ideas of [9]. See also Lin-Riviere [11], Li-Tian [12] and Riviere [17], Tian [21]. Finally, Theorem 1.2 also covers the sign-changing case.

As an application we can improve Pacard's result when $n-2 \frac{p+1}{p-1}$ is not an integer, that is

Theorem 1.3. Let $u$ be a stationary solution to (1.1), then the Hausdorff dimension of $\mathcal{S}(u)$ is no more than $n-m$. Moreover, if $p<\frac{n+1}{n-3}$, $\mathcal{S}(u)$ is a discrete set.

As another application, we consider a problem studied by Dancer [3]. Given a smooth bounded domain $\Omega \subset \mathbb{R}^{n}$, consider the problem

$$
\begin{cases}-\Delta u=\lambda(1+u)^{p} & \text { in } \Omega \\ u>0 & \text { in } \Omega \\ u=0 & \text { on } \partial \Omega\end{cases}
$$

where $p>\frac{n+2}{n-2}, \lambda>0$.

In [3], Dancer proved the existence of a family of positive solutions $(\lambda(s), u(s))$ such that $\|u(s)\|_{L^{\infty}(\Omega)} \rightarrow+\infty$ while $\lambda(s)$ bounded (from below and above). If $\Omega$ is star-shaped, $u(s)$ are uniformly bounded in 
$H^{1}(\Omega)$ and $\lambda(s)$ is uniformly bounded from below and also above. Furthermore Dancer showed that under the assumptions that $\Omega$ is convex and possesses $n$-axis of symmetries and that $\frac{n+2}{n-2}<p<\frac{n+1}{n-3}$, then the sequence $(\lambda(s), u(s))$ converges to $\left(\lambda_{*}, u_{*}\right)$ in $\mathbb{R} \times C_{\text {loc }}^{k}(\Omega \backslash\{0\})$ where $u^{*}$ is a weak solution of (1.6) with only singularity at the origin.

In the following we remove the symmetry assumption of Dancer.

Theorem 1.4. If $\frac{n+2}{n-2}<p<\frac{n+1}{n-3}$ and $\Omega$ is convex, then given any sequence $\left(\lambda_{i}, u_{i}\right)$ in this family,

- There exists a subsequence such that $\lambda_{i} \rightarrow \lambda_{*}, u_{i} \rightarrow u_{*}$ strongly in $H^{1}(\Omega)$;

- $u_{*}$ is a stationary $H^{1}$ weak solution of (1.6), and it is smooth outside finitely many points $x_{i} \in \Omega, 1 \leq i \leq K$;

- For any $k, u_{i}$ converges to $u_{*}$ in $C_{l o c}^{k}\left(\Omega \backslash \cup_{i=1}^{K}\left\{x_{i}\right\}\right)$.

The convexity is used to guarantee that $u_{i}$ are smooth near $\partial \Omega$ (uniformly in $i$ ). This can be proved by the moving plane method. By this near boundary regularity we see the blow up locus can only appear in the interior of $\Omega$, thus we can apply Theorem 1.2 and Theorem 1.3.

The organization of the paper is as follows: In Section 2, we collect some basic estimates including the monotonicity formula and $\epsilon$ regularity. In Section 3 we give the basic Hausdorff measure estimate. Then in Section 4 we consider the case where $2 \frac{p+1}{p-1}$ is not an integer and carry out the important dimension reduction technique to prove Theorem 1.3. The remaining part is devoted to the analysis of the case when $2 \frac{p+1}{p-1}$ is an integer. We construct the bubbling sequence in Section 5. In Section 6 we give a quantization of the density function. Finally we discuss the stationary property of the blow-up locus.

Acknowledgment. The second author thanks Professors Juan Davila, Manuel del Pino and Fanghua Lin for stimulating discussions. The research of Wei is partially supported by a GRF from RGC of Hong Kong and an NSERC of Canada.

\section{Preliminary Analysis}

We collect some preliminary analysis in this section. The basic tool used in this paper is the following monotonicity formula.

Theorem 2.1. For any $B_{R}(x) \subset B_{1}$ and $r \in(0, R)$,

$$
E(r ; x, u):=\frac{r^{-n+2 \frac{p+1}{p-1}}}{p+3} \int_{B_{r}(x)}\left(\frac{p-1}{2}|\nabla u|^{2}+\frac{p-1}{p+1} u^{p+1}\right)
$$




$$
+\frac{1}{p+3} \frac{d}{d r}\left[r^{-n+2 \frac{p+1}{p-1}} \int_{\partial B_{r}(x)} u^{2}\right]
$$

is nondecreasing in $r$. Moreover, if $E(r ; x, u) \equiv$ const. in $(0, R)$, then $u$ is homogeneous with respect to $x$

$$
u(x+\lambda y)=\lambda^{-\frac{2}{p-1}} u(x+y), \quad y \in B_{R}(x), \lambda \in(0,1) .
$$

Proof. This follows directly from Pacard [14]. In fact, by the proof in [14], we have

$$
\frac{d}{d r} E(r ; x, u)=c(n, p) r^{2 \frac{p+1}{p-1}-n} \int_{\partial B_{r}(x)}\left(\frac{\partial u}{\partial r}+\frac{2}{p-1} r^{-1} u\right)^{2} \geq 0 .
$$

This also gives the homogeneity of $u$ when $E \equiv$ const..

An equivalent form is

$$
E(r ; x, u)=r^{-n+2 \frac{p+1}{p-1}} \int_{B_{r}(x)}\left(\frac{|\nabla u|^{2}}{2}-\frac{|u|^{p+1}}{p+1}\right)+r^{-n+2 \frac{p+1}{p-1}-1} \int_{\partial B_{r}(x)} \frac{u^{2}}{p-1} .
$$

Next we recall the $\varepsilon$-regularity theorem, which was proved in [15]. (See Proposition 2 there. Although the result was only stated for positive solutions, its proof also holds for sign-changing solutions after suitable modifications.)

Theorem 2.2. There exist two constants $\varepsilon_{0}, C>0$, depending only on $p$ and $n$, such that if $u$ is a stationary weak solution of (1.1) in $B_{2 R}$, satisfying

$$
R^{2 \frac{p+1}{p-1}-n} \int_{B_{R}} \frac{p-1}{2}|\nabla u|^{2}+\frac{p-1}{p+1}|u|^{p+1} \leq \varepsilon_{0}
$$

then

$$
\sup _{B_{R / 2}}\left(R^{2}\left|\nabla^{2} u\right|+R|\nabla u|+|u|\right) \leq C R^{-\frac{2}{p-1}} .
$$

In the proof if we tract the dependence of $C$ on $\varepsilon$ carefully, we can show that as $\varepsilon_{0} \rightarrow 0, C\left(\varepsilon_{0}\right) \rightarrow 0$.

The following is a technical result, which will be used in the latter part of this paper to treat the boundary term in the monotonicity formula $E(r ; x, u)$.

Lemma 2.3. If $u \in H^{1}\left(B_{1}\right)$, then for any $s \in[0, n-1)$, the set

$$
E:=\left\{x: \limsup _{r \rightarrow 0} r^{-s} \int_{\partial B_{r}(x)} u^{2}>0\right\}
$$

has zero $H^{s+1}$ measure. 
Proof. Note that by the trace theorem for $H^{1}$ space, $\int_{\partial B_{r}(x)} u^{2}$ is well defined for every $\partial B_{r}(x) \subset B_{1}$.

We claim that $E$ belongs to the set

$$
\left\{x: \limsup _{r \rightarrow 0} r^{-s-1} \int_{B_{r}(x)} u^{2}+|\nabla u|^{2}>0\right\} .
$$

Indeed, if

$$
\limsup _{r \rightarrow 0} r^{-s-1} \int_{B_{r}(x)} u^{2}+|\nabla u|^{2}=0,
$$

then for any $r$, there exists $t_{0} \in(r / 2, r)$ such that

$$
\int_{\partial B_{t_{0}}(x)} u^{2}=o\left(r^{s}\right) .
$$

Next by direct differentiating in $r$, we see

$$
\begin{aligned}
& r^{1-n} \int_{\partial B_{r}(x)} u^{2} \\
\leq & t_{0}^{1-n} \int_{\partial B_{t_{0}}(x)} u^{2}+2 \int_{B_{r}(x) \backslash B_{t_{0}}(x)}|y-x|^{1-n} u \frac{(y-x) \cdot \nabla u}{|y-x|} \\
\leq & t_{0}^{1-n} \int_{\partial B_{t_{0}}(x)} u^{2}+2^{n} r^{1-n}\left(\int_{B_{r}(x) \backslash B_{t_{0}}(x)} u^{2}\right)^{\frac{1}{2}}\left(\int_{B_{r}(x) \backslash B_{t_{0}}(x)}|\nabla u|^{2}\right)^{\frac{1}{2}} \\
= & o\left(r^{s+1-n}\right) .
\end{aligned}
$$

That is, for $r \rightarrow 0$,

$$
\int_{\partial B_{r}(x)} u^{2}=o\left(r^{s}\right)
$$

This proves the claim, and then by [10, Lemma 2.1.1] we get the measure estimate.

The next result is Lemma 4 in [14].

Lemma 2.4. There exists a constant $C>0$ depending only on $p$ and $n$, such that for a stationary solution $u$ in $B_{1}$, for any $x \in B_{1 / 4}$ and $r \in(0,1 / 4)$,

$$
r^{2 \frac{p+1}{p-1}-n} \int_{B_{r}(x)} \frac{p-1}{2}|\nabla u|^{2}+\frac{p-1}{p+1}|u|^{p+1} \leq C E(2 r ; x, u) .
$$

By the monotonicity formula we have 
Corollary 2.5. There exists a constant $C>0$ depending only on $p$ and $n$, such that for any stationary solution $u$ in $B_{1}$, for any $x \in B_{1 / 2}$ and $r \in(0,1 / 8)$,

$r^{2 \frac{p+1}{p-1}-n} \int_{B_{r}(x)}|\nabla u|^{2}+|u|^{p+1} \leq C\left(\int_{B_{1}}|\nabla u|^{2}+|u|^{p+1}+\left(\int_{B_{1}}|u|^{p+1}\right)^{\frac{2}{p+1}}\right)$.

Proof. For any $x \in B_{1 / 2}$,

$$
\begin{aligned}
E\left(\frac{1}{4} ; x, u\right) & \leq 4 \int_{\frac{1}{4}}^{\frac{1}{2}} E(\rho ; x, u) d \rho \\
& \leq C(n, p)\left(\int_{B_{1}}|\nabla u|^{2}+|u|^{p+1}+\int_{B_{1}} u^{2}\right) \\
& \leq C(n, p)\left(\int_{B_{1}}|\nabla u|^{2}+|u|^{p+1}+\left(\int_{B_{1}}|u|^{p+1}\right)^{\frac{2}{p+1}}\right) .
\end{aligned}
$$

Then we can apply the previous lemma to get the claim.

Define

$$
\begin{gathered}
\mu_{1, i}=\frac{1}{2}\left|\nabla u_{i}\right|^{2} d x \rightarrow \mu_{1}=\frac{1}{2}|\nabla u|^{2} d x+\nu_{1}, \\
\mu_{2, i}=\frac{1}{p+1}\left|u_{i}\right|^{p+1} d x \rightarrow \mu_{2}=\frac{1}{p+1}|u|^{p+1} d x+\nu_{2} .
\end{gathered}
$$

Hence we have $\mu_{i}=(p-1)\left(\mu_{1, i}+\mu_{2, i}\right), \mu=(p-1)\left(\mu_{1}+\mu_{2}\right)$ and $\nu=(p-1)\left(\nu_{1}+\nu_{2}\right)$.

We have the following energy partition between $\nu_{1}$ and $\nu_{2}$.

Lemma 2.6. $2 \nu_{1}=(p+1) \nu_{2}$.

Proof. Because $u_{i} \in H^{1}\left(B_{2}\right) \cap L^{p+1}\left(B_{2}\right)$, by testing the equation (1.1) with $u_{i} \eta^{2}$, where $\eta \in C_{0}^{\infty}\left(B_{2}\right)$, we get

$$
\int_{B_{2}}\left|\nabla u_{i}\right|^{2} \eta^{2}-\left|u_{i}\right|^{p+1} \eta^{2}=\int_{B_{2}} u_{i}^{2} \Delta \frac{\eta^{2}}{2}
$$

By taking $i \rightarrow+\infty$, and noting that $u_{i}$ converges to $u$ strongly in $L^{2}\left(B_{2}\right)$, we get

$$
\int_{B_{2}}\left(|\nabla u|^{2} \eta^{2}-|u|^{p+1} \eta^{2}\right)+\int_{B_{2}} 2 \eta^{2} d \nu_{1}-(p+1) \eta^{2} d \nu_{2}=\int_{B_{2}} u^{2} \Delta \frac{\eta^{2}}{2} .
$$


On the other hand, since $u \in H^{1}\left(B_{2}\right) \cap L^{p+1}\left(B_{2}\right)$ is an $L^{1}$ weak solution to (1.1), by choosing test functions in the form $u^{M} \eta^{2}$, where $u^{M}=$ $\max \{\min \{u, M\},-M\}$, and then letting $M \rightarrow+\infty$, we also have

$$
\int_{B_{2}}|\nabla u|^{2} \eta^{2}-|u|^{p+1} \eta^{2}=\int_{B_{2}} u^{2} \Delta \frac{\eta^{2}}{2} .
$$

Substituting these into (2.3), we see

$$
\int_{B_{2}} 2 \eta^{2} d \nu_{1}-(p+1) \eta^{2} d \nu_{2}=0
$$

Since $\eta$ can be chosen arbitrarily, this gives the claim.

\section{Analysis of the BlOW up locus}

In this section we use notations as in Theorem 1.2. Define

$$
\widetilde{\Sigma}=\cap_{0<r<1}\left\{x \in B_{1}: \limsup _{i \rightarrow \infty} r^{-n+2 \frac{p+1}{p-1}} \mu_{i}\left(B_{r}(x)\right) \geq \frac{\varepsilon_{0}}{2}\right\} .
$$

Below we will show that this coincides with $\Sigma$ defined in the introduction.

Lemma 3.1. $\widetilde{\Sigma}$ is a closed set. For any domain $\Omega \subset \subset B_{1} \backslash \widetilde{\Sigma}$ and $k>0, u_{i}$ converges to $u$ in $C^{k}(\Omega)$.

Proof. By definition, if $x_{0}$ does not belong to $\widetilde{\Sigma}$, there exists an $r_{0}>0$ such that, for all $i$ large,

By Theorem 2.2,

$$
r_{0}^{2 \frac{p+1}{p-1}-n} \mu_{i}\left(B_{r_{0}}(x)\right) \leq \varepsilon_{0} .
$$

$$
\sup _{B_{r_{0} / 2}\left(x_{0}\right)}\left|u_{i}\right| \leq C r_{0}^{-\frac{2}{p-1}} .
$$

Then standard elliptic estimates show that $u_{i}$ are uniformly bounded in $C^{k+1}\left(B_{r_{0} / 4}\left(x_{0}\right)\right)$ for any $k$. This then implies that for any $r \in\left(0, r_{0} / 4\right)$,

$$
\mu_{i}\left(B_{r}\left(x_{0}\right)\right) \leq C r^{2 \frac{p+1}{p-1}} .
$$

Then we get an $r_{1}>0$, which is independent of $i$, such that $B_{r_{1}}\left(x_{0}\right) \cap$ $\widetilde{\Sigma}=\emptyset$. So $\widetilde{\Sigma}$ is relatively closed.

Since $u_{i}$ converges to $u$ in $L^{2}\left(B_{r_{1}}\left(x_{0}\right)\right), u_{i}$ also converges to $u$ in $C^{k}\left(B_{r_{1}}\left(x_{0}\right)\right)$.

From this proof we see

Corollary 3.2. $u$ is smooth outside $\widetilde{\Sigma}$. That is, $\mathcal{S}(u) \subset \widetilde{\Sigma}$.

Lemma 3.1 also shows that $u_{i}$ converges strongly to $u$ in $H_{l o c}^{1} \cap L_{l o c}^{p+1}$ outside $\widetilde{\Sigma}$. Hence by the definition of $\nu$ we obtain 
Corollary 3.3. $\operatorname{spt}(\nu) \subset \widetilde{\Sigma}$.

Since we will encounter many times the weak convergence of positive Radon measures. The following facts may be helpful to keep in mind.

(1) For a positive Radon measure $\mu$ defined in $B_{1}$, except a countable set of $r \in(0,1)$,

$$
\mu\left(\partial B_{r}\right):=\lim _{h \rightarrow 0} \mu\left(B_{r+h} \backslash \overline{B_{r-h}}\right)=0 .
$$

(2) Assume that a sequence of positive Radon measures $\mu_{i}$ converges weakly to $\mu$. Then for any open bounded set $\Omega$,

$$
\begin{aligned}
& \liminf _{i \rightarrow+\infty} \mu_{i}(\Omega) \geq \mu(\Omega), \\
& \limsup _{i \rightarrow+\infty} \mu_{i}(\bar{\Omega}) \leq \mu(\bar{\Omega}) .
\end{aligned}
$$

(3) Combining the previous two points, we see for a.a. $r>0$,

$$
\lim _{i \rightarrow+\infty} \mu_{i}\left(B_{r}\right)=\mu\left(B_{r}\right) .
$$

Lemma 3.4. $H^{n-2 \frac{p+1}{p-1}}\left(\widetilde{\Sigma} \cap B_{1}\right)<+\infty$.

Proof. For any $x \in \widetilde{\Sigma}$ and $r \in(0,1)$, if $\mu\left(\partial B_{r}(x)\right)=0$, then

$$
\mu\left(B_{r}\right)=\lim _{i \rightarrow+\infty} \mu_{i}\left(B_{r}\right) .
$$

So by the definition of $\widetilde{\Sigma}$, we have

$$
r^{-n+2 \frac{p+1}{p-1}} \mu\left(B_{r}(x)\right) \geq c \varepsilon_{0} .
$$

If $\mu\left(\partial B_{r}(x)\right) \neq 0$, we can take an increasing sequence $r_{i} \rightarrow r$ with $\mu\left(\partial B_{r_{i}}(x)\right)=0$, so that (3.1) holds for $r_{i}$. Then by letting $i \rightarrow+\infty$, we see (3.1) also holds for such $r$, and hence for any $r>0$.

The measure estimate can be proved by the Vitali covering theorem, see [8, Theorem 3.2].

Remark 3.5. In fact the proof shows that, for any $x \in \widetilde{\Sigma} \cap B_{1}$ and $r \in(0,1 / 2)$,

$$
H^{n-2 \frac{p+1}{p-1}}\left(B_{r} \cap \widetilde{\Sigma}\right) \leq C(M) r^{n-2 \frac{p+1}{p-1}} .
$$

Concerning the upper bound, we have

Lemma 3.6. For any $x \in \widetilde{\Sigma}$ and $r \in(0,1)$,

$$
\mu\left(B_{r}(x)\right) \leq C(M) r^{n-2 \frac{p+1}{p-1}} .
$$


Proof. By (2.2), for any $i>0, x \in B_{1}$ and $r \in(0,1)$,

$$
r^{-n+2 \frac{p+1}{p-1}} \mu_{i}\left(B_{r}(x)\right) \leq C\left(\int_{B_{2}}\left|\nabla u_{i}\right|^{2}+\left|u_{i}\right|^{p+1}+\left(\int_{B_{1}}\left|u_{i}\right|^{p+1}\right)^{\frac{2}{p+1}}\right) .
$$

Then (3.2) follows frow the weak convergence of $\mu_{i}$ to $\mu$.

In particular, $\mu\left\lfloor_{\Sigma}\right.$ is absolutely continuous with respect to $\left.H^{n-2 \frac{p+1}{p-1}}\right|_{\widetilde{\Sigma}}$. However we can show

Lemma 3.7. $\mu \mathrm{L}_{\widetilde{\Sigma}}=\nu$.

Proof. Because $u \in H^{1}\left(B_{1}\right) \cap L^{p+1}\left(B_{1}\right)$, by [10, Lemma 2.1.1], the set

$$
\left\{x \in B_{1}: \limsup _{r \rightarrow 0} r^{-n+2 \frac{p+1}{p-1}} \int_{B_{r}(x)}|\nabla u|^{2}+|u|^{p+1}>0\right\}
$$

has zero $n-2(p+1) /(p-1)$ Hausdorff dimensional measure. This means, for $H^{n-2(p+1) /(p-1)}-$ a.a. $x \in \widetilde{\Sigma}$,

$$
\limsup _{r \rightarrow 0} r^{-n+2 \frac{p+1}{p-1}} \int_{B_{r}(x)}|\nabla u|^{2}+|u|^{p+1}=0 .
$$

Because $H^{n-2 \frac{p+1}{p-1}}(\widetilde{\Sigma})<+\infty$, the measure $\left(|\nabla u|^{2}+|u|^{p+1}\right) d x$ restricted to $\widetilde{\Sigma}$ is zero. So $\mu \mathrm{L}_{\widetilde{\Sigma}}=\nu$.

This result, combined with (3.1) and (3.2), implies that $\nu$ and $H^{n-2 \frac{p+1}{p-1}}\left\lfloor_{\widetilde{\Sigma}}\right.$ are mutually continuous with respect to each other. Together with Lemma 3.1 and (3.1), this lemma also implies that, when $\nu \neq 0$, the support of $\nu$ is exactly $\widetilde{\Sigma}$. Since we always have $\mathcal{S}(u) \subset \widetilde{\Sigma}$, we see in this case $\Sigma=\widetilde{\Sigma}$.

If $\nu=0$, the proof of Lemma 3.7 implies that for $H^{n-2 \frac{p+1}{p-1}}$-a.a. $x \in \Sigma$,

$$
\limsup _{r \rightarrow 0} r^{2 \frac{p+1}{p-1}-n} \mu\left(B_{r}(x)\right)=0 .
$$

Combining this with Lemma 3.4, we see

$$
H^{n-2 \frac{p+1}{p-1}}(\widetilde{\Sigma})=0 .
$$

In this case, we still have

Lemma 3.8. If $\nu=0, \widetilde{\Sigma}=\mathcal{S}(u)=\Sigma$.

Proof. The assumption that $\nu=0$ implies the strong convergence of $u_{i}$ in $H_{l o c}^{1} \cap L_{l o c}^{p+1}$. If $x_{0}$ does not belong to $\mathcal{S}(u)$, by definition $u$ is smooth in an open ball $B_{r_{0}}\left(x_{0}\right)$. Then there exists another $r_{1}<r_{0}$ so that

$$
r_{1}^{2 \frac{p+1}{p-1}-n} \int_{B_{r_{1}}\left(x_{0}\right)} \frac{p-1}{2}|\nabla u|^{2}+\frac{p-1}{p+1}|u|^{p+1} \leq \frac{\varepsilon_{0}}{4} .
$$


By the strong convergence of $u_{i}$ in $H_{l o c}^{1} \cap L_{l o c}^{p+1}$, for all $i$ large,

$$
r_{1}^{2 \frac{p+1}{p-1}-n} \int_{B_{r_{1}}\left(x_{0}\right)} \frac{p-1}{2}\left|\nabla u_{i}\right|^{2}+\frac{p-1}{p+1}\left|u_{i}\right|^{p+1}<\frac{\varepsilon_{0}}{2} .
$$

Thus we can argue as in the proof of Lemma 3.1 to show that $x_{0}$ does not belong to $\widetilde{\Sigma}$. This gives $\widetilde{\Sigma} \subset \mathcal{S}(u)$, and the other direction has already been given in Corollary 3.2.

This finishes the proof of the first claim in Theorem 1.2. Next we study the structure of $\Sigma$.

Lemma 3.9. For $H^{n-2 \frac{p+1}{p-1}}-$ a.a. $x \in \Sigma$,

$$
\Theta(x):=\lim _{r \rightarrow 0} r^{-n+2 \frac{p+1}{p-1}} \nu\left(B_{r}(x)\right) \in\left(\frac{\varepsilon_{0}}{2}, C(M)\right),
$$

exists.

Proof. Fix a point in $\Sigma$, and without loss of generality, assume it is 0 .

By (2.1), for any $0<r_{1}<r_{2}<1$,

$$
E\left(r_{2} ; 0, u_{i}\right) \geq E\left(r_{1} ; 0, u_{i}\right) .
$$

Here we use the second formulation of $E(r ; x, u)$.

By the weak convergence of $u_{i}$ in $H_{l o c}^{1}$ and the trace theorem, for any ball $B_{r}(x)$,

$$
\lim _{i \rightarrow+\infty} \int_{\partial B_{r}(x)} u_{i}^{2}=\int_{\partial B_{r}(x)} u^{2} .
$$

For a.a. $r \in(0,1), \mu\left(\partial B_{r}(x)\right)=0$. For such $r$, we have

$$
\mu\left(B_{r}(x)\right)=\lim _{i \rightarrow \infty} \mu_{i}\left(B_{r}(x)\right) .
$$

The same claims also hold for $\nu, \nu_{1}$ and $\nu_{2}$. If $r_{1}, r_{2}$ satisfy these conditions, then passing to the limit in the monotonicity formula for $u_{i}$ we obtain

$E\left(r_{2} ; 0, u\right)+r_{2}^{2 \frac{p+1}{p-1}-n}\left(\nu_{1}-\nu_{2}\right)\left(B_{r_{2}}\right) \geq E\left(r_{1} ; 0, u\right)+r_{1}^{2 \frac{p+1}{p-1}-n}\left(\nu_{1}-\nu_{2}\right)\left(B_{r_{2}}\right)$.

Note that by Lemma 2.6, $\nu_{1}-\nu_{2}=\frac{p-1}{2} \nu_{2}=\frac{1}{p+3} \nu$.

If $\mu\left(\partial B_{r_{1}}\right) \neq 0$ or $\mu\left(\partial B_{r_{2}}\right) \neq 0$, we can take $\bar{r}_{1}>r_{1}, \bar{r}_{2}<r_{2}$, with $\bar{r}_{1}<\bar{r}_{2}$ and $\mu\left(\partial B_{\bar{r}_{1}}\right)=\mu\left(\partial B_{\bar{r}_{2}}\right)=0$, so that

$E\left(\bar{r}_{2} ; 0, u\right)+\bar{r}_{2}^{2 \frac{p+1}{p-1}-n}\left(\nu_{1}-\nu_{2}\right)\left(B_{\bar{r}_{2}}\right) \geq E\left(\bar{r}_{1} ; 0, u\right)+\bar{r}_{1}^{2 \frac{p+1}{p-1}-n}\left(\nu_{1}-\nu_{2}\right)\left(B_{\bar{r}_{1}}\right)$.

For any $\varepsilon>0$, we can choose $\bar{r}_{2}$ close to $r_{2}$ so that

$$
\left(\nu_{1}-\nu_{2}\right)\left(B_{\bar{r}_{2}}\right) \geq\left(\nu_{1}-\nu_{2}\right)\left(B_{r_{2}}\right)-\varepsilon
$$


Then by noting that $E(r ; 0, u)$ is continuous in $r$, and $\left(\nu_{1}-\nu_{2}\right)\left(B_{\bar{r}_{1}}\right) \geq$ $\left(\nu_{1}-\nu_{2}\right)\left(B_{r_{1}}\right)$, we can let $\bar{r}_{1} \rightarrow r_{1}, \bar{r}_{2} \rightarrow r_{2}$ to get (3.4).Thus (3.4) holds for any $0<r_{1}<r_{2}<1$.

By Lemma 3.6, we directly get a lower bound for

$$
\bar{E}(r):=E(r ; 0, u)+\frac{1}{p+3} r^{2 \frac{p+1}{p-1}-n} \nu\left(B_{r}\right) \geq-C(M) .
$$

By the monotonicity of $\bar{E}(r)$, we can use the same method as in the proof of Corollary 2.5 to obtain an upper bound for $\bar{E}(r)$.

Then once again by the monotonicity, the limit

$$
\lim _{r \rightarrow 0}\left(E(r ; 0, u)+\frac{1}{p+3} r^{2 \frac{p+1}{p-1}-n} \nu\left(B_{r}\right)\right)
$$

exists.

Now we assume that (3.3) holds at 0 , which is true $H^{n-2 \frac{p+1}{p-1}}$ a.e. in $\Sigma$. By Lemma 2.3, we can also assume that at this point

$$
\lim _{r \rightarrow 0} r^{2 \frac{p+1}{p-1}-n-1} \int_{\partial B_{r}(x)} u^{2}=0 .
$$

With this choice, at this point

$$
\lim _{r \rightarrow 0} E(r ; 0, u)=0 .
$$

Thus the limit

$$
\Theta(0)=\lim _{r \rightarrow 0} r^{2 \frac{p+1}{p-1}-n} \nu\left(B_{r}\right)
$$

exists.

Finally, the upper bound of $\Theta$ is a direct consequence of (3.2). Concerning the lower bound, we can use (3.3) and (3.5) again to see that, as $r \rightarrow 0$,

$$
\begin{aligned}
\Theta(0) & =r^{2 \frac{p+1}{p-1}-n} \nu\left(B_{r}\right)+o(1) \\
& =r^{2 \frac{p+1}{p-1}-n} \mu\left(B_{r}\right)+o(1) \\
& \geq \frac{\varepsilon_{0}}{2}+o(1) .
\end{aligned}
$$

Here $o(1)$ goes to 0 as $r \rightarrow 0$.

By the Radon-Nikodym theorem, we get

Corollary 3.10. $\nu=\Theta(x) H^{n-m}\left\lfloor_{\Sigma}\right.$.

From the proof we also get

Corollary 3.11. For any $x \in B_{1}$ and $r \in(0,1 / 2)$,

$$
\bar{E}(r ; x):=E(r ; x, u)+\frac{1}{p+3} r^{2 \frac{p+1}{p-1}-n} \nu\left(B_{r}(x)\right)
$$


is non-decreasing in $r$.

By Marstrand theorem ([10, Theorem 1.3.12] and [13]), if $n-2 \frac{p+1}{p-1}$ is not an integer, we must have $\nu=0$. This then implies that

$\left(\frac{p-1}{2}\left|\nabla u_{i}\right|^{2}+\frac{p-1}{p+1}\left|u_{i}\right|^{p+1}\right) d x \rightarrow\left(\frac{p-1}{2}\left|\nabla u_{i}\right|^{2}+\frac{p-1}{p+1}\left|u_{i}\right|^{p+1}\right) d x$.

Because $u_{i} \rightarrow u$ weakly in $H_{l o c}^{1} \cap L_{l o c}^{p+1}, u_{i} \rightarrow u$ strongly in $H_{l o c}^{1} \cap L_{l o c}^{p+1}$. Then the stationary condition for $u_{i}$ can be passed to the limit, so $u$ also satisfies the stationary condition. This finishes the proof of the second claim of Theorem 1.2.

\section{THE DIMENSION REDUCTION}

In this section we consider the partial regularity for a stationary solution $u$ to (1.1), where $n-2 \frac{p+1}{p-1}$ is not an integer. A crucial point is the fact we have just established: weak convergent solutions of (1.1) also converges strongly in $H_{l o c}^{1} \cap L_{l o c}^{p+1}$.

Pacard's partial regularity result ([14]) says that the singular set of $u, \mathcal{S}(u)$ is a closed set satisfying

$$
H^{n-2 \frac{p+1}{p-1}}(\mathcal{S}(u))=0 .
$$

In particular, $\operatorname{dim}(\mathcal{S}(u)) \leq n-2 \frac{p+1}{p-1}$. We will use Federer's dimension reduction principle to reduce this dimension to $n-m$, the integer part of $n-2 \frac{p+1}{p-1}$.

By the monotonicity of $E(r ; x, u)$, we can define the density function

$$
\Theta(x, u)=\lim _{r \rightarrow 0^{+}} E(r ; x, u) .
$$

By [14, Lemma 2 and Lemma 3], we have

Lemma 4.1. $\Theta(x, u) \geq 0$ is an upper-continuous function.

Next we have the following characterization of singular points.

Proposition 4.2. There exists a constant $\varepsilon_{1}$ depending only on $n$ and $p$, such that for any stationary weak solution $u$ of $(1.1), x \in \mathcal{S}(u)$ if and only if $\Theta(x, u) \geq \varepsilon_{1}$.

Proof. If $x$ is in the regular set of $u$, there exists an $r_{0}>0$ such that $u$ is smooth in $B_{r_{0}}(x)$. Then there exists a constant $C$ such that for every $r<r_{0}$,

$$
\int_{B_{r}(x)}|\nabla u|^{2}+|u|^{p+1} \leq C r^{n}, \quad \int_{\partial B_{r}(x)} u^{2} \leq C r^{n-1} .
$$


Substituting this into the second formulation of $E(r ; x, u)$ we get

$$
E(r ; x, u) \leq C r^{2 \frac{p+1}{p-1}} .
$$

Thus $\Theta(x, u)=0$.

On the other hand, for $x \in \mathcal{S}(u)$, by Theorem 2.2 and Lemma 2.4, there exists a universal constant $\varepsilon_{1}>0$ such that for any $r>0$,

$$
E(r ; x, u) \geq \varepsilon_{1} \text {. }
$$

By definition, we get $\Theta(x ; u) \geq \varepsilon_{1}$.

Assume $0 \in \mathcal{S}(u)$. For $\lambda \rightarrow 0$, define the blow up sequence

$$
u^{\lambda}(x)=\lambda^{\frac{2}{p-1}} u(\lambda x) .
$$

By rescaling Lemma 2.4, for any $x \in B_{\lambda^{-1} / 2}$ and $r \in\left(0, \lambda^{-1} / 2\right)$,

$$
\int_{B_{r}(x)}\left|\nabla u^{\lambda}\right|^{2}+\left|u^{\lambda}\right|^{p+1} \leq C(M) r^{n-2 \frac{p+1}{p-1}} .
$$

Here $C(M)$ is a constant independent of $\lambda \rightarrow 0$.

By Theorem 1.2, we can subtract a subsequence $\lambda_{i} \rightarrow 0$ such that $u_{i}:=u^{\lambda_{i}}$ converges strongly to a stationary solution $u_{\infty}$ in $H_{l o c}^{1}\left(\mathbb{R}^{n}\right) \cap$ $L_{l o c}^{p+1}\left(\mathbb{R}^{n}\right)$.

By the weak convergence of $u_{i}$ in $H_{l o c}^{1}\left(\mathbb{R}^{n}\right)$ and the trace theorem, for any $r>0$ and $x \in \mathbb{R}^{n}$,

$$
\int_{\partial B_{r}(x)} u_{\infty}^{2}=\lim _{i \rightarrow+\infty} \int_{\partial B_{r}(x)} u_{i}^{2} .
$$

Then we get

$$
E\left(r ; 0, u_{\infty}\right)=\lim _{i \rightarrow+\infty} E\left(r ; 0, u_{i}\right) .
$$

On the other hand, a direct scaling shows

$$
E\left(r ; 0, u_{i}\right)=E\left(\lambda_{i} r ; 0, u\right) .
$$

By the monotonicity of $E(r ; 0, u)$, we obtain

$$
E\left(r ; 0, u_{\infty}\right) \equiv \lim _{r \rightarrow 0} E(r ; 0, u)=\Theta(0, u), \forall r>0 .
$$

By Theorem 2.1, $u_{\infty}$ is homogeneous, that is, for any $\lambda>0$,

$$
u_{\infty}(\lambda x)=\lambda^{-\frac{2}{p-1}} u_{\infty}(x) \text { a.e. in } \mathbb{R}^{n} .
$$

In particular, the singular set $\mathcal{S}\left(u_{\infty}\right)$ is a cone, that is,

$$
\lambda \mathcal{S}\left(u_{\infty}\right)=\mathcal{S}\left(u_{\infty}\right), \quad \forall \lambda>0 .
$$

By Theorem 1.2 we have

Lemma 4.3. For any $\varepsilon>0$, if $i$ large, $\mathcal{S}\left(u_{i}\right) \cap B_{1}$ lies in an $\varepsilon-$ neighborhood of $\mathcal{S}\left(u_{\infty}\right) \cap B_{1}$. 
Proof. Because $n-2 \frac{p+1}{p-1}$ is not an integer, the blow up locus $\Sigma=\mathcal{S}\left(u_{\infty}\right)$. Since $u_{i}$ converges to $u_{\infty}$ in any $C^{k}$ topology away from $\Sigma$, for all $i$ large, $u_{i}$ is smooth outside the $\varepsilon$-neighborhood of $\mathcal{S}\left(u_{\infty}\right)$, and by this the claim can be seen.

The following result is the key step to apply Federer's dimension reduction principle. The proof can be found in [22] (cf. Lemma 3.2 therein. The proof only uses the validation of the monotonicity formula, Theorem 2.1).

Lemma 4.4. Given a stationary weak solution $u$ of $(1.1)$ on $\mathbb{R}^{n}$, assume that $u$ is homogeneous, that is, $\forall \lambda>0$,

$$
u(\lambda x)=\lambda^{-\frac{2}{p-1}} u(x) .
$$

Then $\forall x \in \mathbb{R}^{n}, \Theta(x, u) \leq \Theta(0, u)$. Moreover, if $\Theta(x, u)=\Theta(0, u)$, then $u$ is invariant in the direction of $x$, i.e. $\forall t \in \mathbb{R}$,

$$
u(y+t x)=u(y), \text { a.e. } y \in \mathbb{R}^{n} .
$$

The last claim means $u$ can viewed as a solution of $(1.1)$ in $\mathbb{R}^{n-1}$. The following result shows that the stationary condition is preserved under this operation.

Lemma 4.5. Let $u=u\left(x_{1}, \cdots, x_{n-1}\right) \in H_{l o c}^{1}\left(\mathbb{R}^{n-1}\right) \cap L_{l o c}^{p+1}\left(\mathbb{R}^{n-1}\right)$ be a weak solution of (1.1) in $\mathbb{R}^{n-1}$. Take $\bar{u}$ to be the trivial extension of $u$ to $\mathbb{R}^{n}$,

$$
\bar{u}\left(x_{1}, \cdots, x_{n}\right)=u\left(x_{1}, \cdots, x_{n-1}\right) .
$$

Then $u$ is stationary if and only if $\bar{u}$ is stationary.

Proof. First assume $\bar{u}$ is stationary but $u$ is not stationary. By definition there exists a vector field $Y \in C_{0}^{\infty}\left(\mathbb{R}^{n-1}, \mathbb{R}^{n-1}\right)$, such that

$$
\int_{\mathbb{R}^{n-1}}\left(\frac{1}{2}|\nabla u|^{2}-\frac{1}{p+1}|u|^{p+1}\right) \operatorname{div} Y-D Y(\nabla u, \nabla u)=\delta>0 .
$$

For any $T$, take a function $\eta_{T} \in C_{0}^{\infty}((-T-1, T+1))$ such that $\eta \equiv 1$ in $(-T, T),\left|\eta^{\prime}\right| \leq 2$. Then

$$
\bar{Y}\left(x_{1}, \cdots, x_{n-1}, x_{n}\right)=Y\left(x_{1}, \cdots, x_{n-1}\right) \eta\left(x_{n}\right)
$$

is a smooth vector field in $\mathbb{R}^{n}$ with compact support. So

$$
\int_{\mathbb{R}^{n}}\left(\frac{1}{2}|\nabla \bar{u}|^{2}-\frac{1}{p+1}|\bar{u}|^{p+1}\right) \operatorname{div} \bar{Y}-D \bar{Y}(\nabla \bar{u}, \nabla \bar{u})=0 .
$$

However, direct calculation shows that this also equals

$$
\int_{\mathbb{R}^{n-1} \times\left\{-T<x_{n}<T\right\}}\left(\frac{1}{2}|\nabla u|^{2}-\frac{1}{p+1}|u|^{p+1}\right) \operatorname{div} Y-D Y(\nabla u, \nabla u)
$$




$$
\begin{aligned}
& +\int_{\mathbb{R}^{n-1} \times\left\{T<\left|x_{n}\right|<T+1\right\}}\left(\frac{1}{2}|\nabla \bar{u}|^{2}-\frac{1}{p+1}|\bar{u}|^{p+1}\right) \operatorname{div} \bar{Y}-D \bar{Y}(\nabla \bar{u}, \nabla \bar{u}) \\
& =2 T \delta+O(1) .
\end{aligned}
$$

Hence if we choose $T$ large we get a contradiction. This proves the stationary condition for $u$.

Now assume $u$ is stationary. For any vector field $\bar{Y} \in C_{0}^{\infty}\left(\mathbb{R}^{n}, \mathbb{R}^{n}\right)$, by noting that $\frac{\partial \bar{u}}{\partial x_{n}}=0$ a.e., we have

$$
\begin{aligned}
& \int_{\mathbb{R}^{n}}\left(\frac{1}{2}|\nabla \bar{u}|^{2}-\frac{1}{p+1}|\bar{u}|^{p+1}\right) \operatorname{div} \bar{Y}-D \bar{Y}(\nabla \bar{u}, \nabla \bar{u}) \\
= & \int_{-\infty}^{+\infty} \int_{\mathbb{R}^{n-1}}\left(\frac{1}{2}|\nabla u|^{2}-\frac{1}{p+1}|u|^{p+1}\right) \sum_{1 \leq i \leq n-1} \frac{\partial \bar{Y}_{i}}{\partial x_{i}}-\sum_{1 \leq i, j \leq n-1} \frac{\partial \bar{Y}_{i}}{\partial x_{j}} \frac{\partial \bar{u}}{\partial x_{i}} \frac{\partial \bar{u}}{\partial x_{j}} \\
& +\int_{\mathbb{R}^{n-1}}\left(\frac{1}{2}|\nabla u|^{2}-\frac{1}{p+1}|u|^{p+1}\right) \times \int_{-\infty}^{+\infty} \frac{\partial \bar{Y}_{n}}{\partial x_{n}} \\
= & 0 .
\end{aligned}
$$

This proves the stationary condition for $\bar{u}$.

When the blow up limit $u_{\infty}$ has a singular point $x_{1} \neq 0$, the next step in Federer's dimension reduction argument is to blow up $u_{\infty}$ once again at $x_{1}$, thus obtaining another homogeneous solution $u_{\infty, 1}$. By Lemma 4.1 and Lemma 4.4, we can show that $u_{\infty, 1}$ is translation invariant along the direction $x_{1}$. Hence we can view it as a solution defined on $\mathbb{R}^{n-1}$, which is also stationary by Lemma 4.5 . Note that this operation also decreases the Hausdorff dimension of its singular set by 1 . We can repeat this step until we get a homogeneous solution defined on $\mathbb{R}^{k}$, which is singular only at the origin 0 . Since by our assumption $p \in\left(\frac{m+2}{m-2}, \frac{m+1}{m-3}\right)$ (in particular, $p$ is subcritical in dimension $m-1$ ), it can be directly verified that $k \geq m$. Roughly speaking, after at most $n-m$ steps, we get a solution with singular set of dimension 0 . Recall that at each step of reduction we decrease the dimension of singular sets by 1 , thus the dimension of $\mathcal{S}(u)$ is at most $n-m$. This proves Theorem 1.3. For a precise treatment of this argument and also the case when $p \in\left(\frac{n+2}{n-2}, \frac{n+1}{n-3}\right)$, we refer to $[18$, Appendix A] and [10, Section 2.3].

\section{The Bubble CONSTRUCTION}

In this section and the following parts of this paper we consider the case where $n-2 \frac{p+1}{p-1}$ is an integer. Denote $m=2 \frac{p+1}{p-1}$, that is, $p$ is the critical Sobolev exponent in $\mathbb{R}^{m}$. 
We first explore the local properties of $\Sigma$ near an arbitrary point, say $0 \in \Sigma$. For any $\lambda>0$, define $\mu^{\lambda}:=L_{\sharp}^{\lambda, 0} \mu$, i.e.

$$
\mu^{\lambda}(A):=\lambda^{2 \frac{p+1}{p-1}-n} \mu(\lambda A) \text { for any Borel set } A .
$$

By (3.2), for any ball $B_{r}$,

$$
\mu^{\lambda}\left(B_{r}\right)=\lambda^{2 \frac{p+1}{p-1}-n} \mu\left(B_{\lambda r}\right) \leq C(M) r^{n-2 \frac{p+1}{p-1}} .
$$

Hence we can subtract a subsequence $\lambda_{j} \rightarrow 0$, so that $\mu^{\lambda_{j}}$ converges weakly to a positive Radon measure $\mu_{0}$ on $\mathbb{R}^{n}$. Note that for a.a. $r>0$, we have $\mu_{0}\left(\partial B_{r}\right)=0$. Then for such $r$, by (3.1),

$$
\mu_{0}\left(B_{r}\right)=\lim _{j \rightarrow+\infty} \mu_{\lambda_{j}}\left(B_{r}\right) \geq c \varepsilon_{0} r^{n-2 \frac{p+1}{p-1}} .
$$

A posterior this holds for all $r>0$ by continuity, not only those $r$ with $\mu_{0}\left(\partial B_{r}\right)=0$. In particular this implies that $\mu_{0} \neq 0$ is nontrivial.

By a rescaling using Remark 3.5, we see for any $\lambda, r>0$,

$$
H^{n-m}\left(B_{r} \cap \Sigma^{\lambda}\right) \leq C(M) r^{n-m} .
$$

where $\Sigma^{\lambda}:=\lambda^{-1} \Sigma$. By the Blaschke Selection Theorem (cf. [10, Theorem 2.1.5]), after further subtracting a subsequence of $\lambda_{j} \rightarrow 0$, we can assume that $\Sigma^{\lambda_{j}}$ converges to a closed set $\Sigma^{0}$.

By Lemma 3.9 and Preiss theorem ([16], see also [10] for a direct proof without using Preiss theorem), $\Sigma$ is countably $(n-m)$-rectifiable. In particular, for $H^{n-m}$-a.a. $x \in \Sigma$, there exists a tangent plane $T$ to $\Sigma$. (This can also be proved directly, see [9, Section 2].) Thus for any $\varepsilon>0$, as $\lambda \rightarrow 0$,

$$
\lambda^{-1}(\Sigma-\{x\}) \cap B_{1} \text { belongs to an } \varepsilon \text { neighborhood of } T \text {. }
$$

Recall that

$$
\Theta(x)=\lim _{r \rightarrow 0} r^{m-n} \nu\left(B_{r}(x)\right), H^{n-m}-\text { a.e. in } \Sigma,
$$

is Borel measurable. We have (for a direct proof see for example [21, Lemma 3.2.2])

Lemma 5.1. $\Theta(x)$ is $H^{n-m}$ approximate continuous at $H^{n-m}-a . a$. $x \in \Sigma$. Here $\Theta(x)$ is $H^{n-m}$ approximate continuous at $x_{0}$ if for any $\varepsilon>0$,

$$
\lim _{r \rightarrow 0} \frac{H^{n-m}\left(\left\{x \in \Sigma \cap B_{r}\left(x_{0}\right):\left|\Theta(x)-\Theta\left(x_{0}\right)\right|>\varepsilon\right\}\right)}{H^{n-m}\left(B_{r}\left(x_{0}\right) \cap \Sigma\right)}=0 .
$$

If 0 is an approximate continuous point of $\Theta$, then by the same proof of $[9$, Lemma 2.1], there exists a tangent plane $T$ of $\Sigma$ at 0 . Without 
loss of generality, assume $T=\mathbb{R}^{n-m} \times\{0\} \subset \mathbb{R}^{n}$. We can also assume that (3.3) holds at 0, i.e.

$$
\lim _{r \rightarrow 0} r^{m-n} \int_{B_{r}}\left(|\nabla u|^{2}+|u|^{p+1}\right)=0 .
$$

In this case $\Sigma^{0}=T$ and $\mu_{0}=\Theta(0) H^{n-m}\left\lfloor_{T}\right.$.

In the following we will always assume that such a base point has been chosen. Denote $x=\left(x^{\prime}, x^{\prime \prime}\right) \in \mathbb{R}^{n-m} \times \mathbb{R}^{m}$, and open balls in $\mathbb{R}^{n-m}\left(\right.$ or $\left.\mathbb{R}^{m}\right)$ by $B_{r}^{\prime}\left(x^{\prime}\right)$ (respectively, $B_{r}^{\prime \prime}\left(x^{\prime \prime}\right)$ ).

For each $\lambda$, the sequence $u_{i}^{\lambda}(x):=\lambda^{\frac{2}{p-1}} u_{i}(\lambda x)$ converges to $u^{\lambda}(x):=$ $\lambda^{\frac{2}{p-1}} u(\lambda x)$ weakly in $H^{1}\left(B_{2 \lambda^{-1}}\right) \cap L^{p+1}\left(B_{2 \lambda^{-1}}\right)$. As $i \rightarrow+\infty$, the measures

$$
\mu_{i}^{\lambda}=L_{\sharp}^{\lambda, 0} \mu_{i} \rightarrow\left(\frac{p-1}{2}\left|\nabla u^{\lambda}\right|^{2}+\frac{p-1}{p+1}\left|u^{\lambda}\right|^{p+1}\right) d x+\nu^{\lambda}=\mu^{\lambda} .
$$

For each $j$, we can choose an $i(j)$ and $R_{j}$ large so that the Levi distance between $\mu_{i(j)}^{\lambda_{j}}\left\lfloor_{B_{R_{j}}}\right.$ and $\nu^{\lambda_{j}}\left\lfloor_{B_{R_{j}}}\right.$ is smaller than $1 / j$. Then by a diagonal argument, we find a sequence of stationary solutions $v_{j}$ to (1.1), satisfying

$\int_{B_{r}(x)}\left|\nabla v_{j}\right|^{2}+\left|v_{j}\right|^{p+1} \leq C(M) r^{n-2 \frac{p+1}{p-1}}$, for all $x \in B_{R_{j} / 2}, r \in\left(0, R_{j} / 2\right)$,

and

$$
\mu_{j}:=\left(\frac{p-1}{2}\left|\nabla v_{j}\right|^{2}+\frac{p-1}{p+1}\left|v_{j}\right|^{p+1}\right) d x \rightarrow \mu_{0}=\Theta(0) H^{n-m}\left\lfloor_{T} .\right.
$$

First we note

Lemma 5.2. In $L_{l o c}^{1}\left(\mathbb{R}^{n}\right)$,

$$
\sum_{k=1}^{n-m}\left|\frac{\partial v_{j}}{\partial x_{k}}\right|^{2} d x \rightarrow 0
$$

Proof. Because

$$
\mu_{j}:=\left(\frac{p-1}{2}\left|\nabla v_{j}\right|^{2}+\frac{p-1}{p+1}\left|v_{j}\right|^{p+1}\right) d x \rightarrow 0 \text { outside } T,
$$

and $T$ is a subspace of $\mathbb{R}^{n}$ with codimension $m \geq 3, v_{j}$ must converge weakly to 0 in $H_{l o c}^{1}\left(\mathbb{R}^{n}\right) \cap L_{l o c}^{p+1}\left(\mathbb{R}^{n}\right)$. By the compact Sobolev embedding theorem, $v_{j}$ converges to 0 in $L_{\text {loc }}^{2}\left(\mathbb{R}^{n}\right)$.

For a.a. $r>0, \mu_{0}\left(\partial B_{r}(0)\right)=0$. By the strong convergence of $v_{j}$ in $L_{l o c}^{2}\left(\mathbb{R}^{n}\right)$, after passing to a subsequence of $j$ again, we also have for 
a.a. $r>0$,

$$
\int_{\partial B_{r}(0)} v_{j}^{2} \rightarrow 0
$$

For such $r$, we have

$$
\begin{aligned}
\mu_{0}\left(B_{r}(0)\right) & =(p+3)\left(\nu_{1}\left(B_{r}(0)\right)-\nu_{2}\left(B_{r}(0)\right)\right) \\
& =\lim _{j \rightarrow+\infty}(p+3) \int_{B_{r}(0)} \frac{\left|\nabla v_{j}\right|^{2}}{2}-\frac{\left|v_{j}\right|^{p+1}}{p+1} .
\end{aligned}
$$

Since $\mu_{0}\left(B_{r}(0)\right) \equiv C r^{n-2 \frac{p+1}{p-1}}$ for some constant $C$, for any generic $0<$ $\sigma<R<+\infty$ (avoiding a countable set),

$$
\lim _{j \rightarrow+\infty} E\left(R ; 0, v_{j}\right)-E\left(\sigma ; 0, v_{j}\right)=0 .
$$

By the monotonicity formula for $v_{j}$, we see

$$
\lim _{j \rightarrow+\infty} \int_{B_{R}(0) \backslash B_{\sigma}(0)} \frac{\left(\frac{y}{|y|} \nabla v_{j}+\frac{2}{p-1}|y|^{-1} v_{j}\right)^{2}}{|y|^{n-m}}=0 .
$$

Because $v_{j}$ converges to 0 in $L_{l o c}^{2}\left(\mathbb{R}^{n}\right)$, after an expansion we obtain

$$
\lim _{j \rightarrow+\infty} \int_{B_{R}(0) \backslash B_{\sigma}(0)}\left|y \cdot \nabla v_{j}\right|^{2}=0 .
$$

Take the standard basis $\xi_{k}$ of $\mathbb{R}^{n-m}, 1 \leq k \leq n-m$. If we choose $R$ and $\sigma$ suitably, the same argument above still works if we replace the center of ball by $\xi_{k}$, which gives

$$
\lim _{j \rightarrow+\infty} \int_{B_{R}\left(\xi_{k}\right) \backslash B_{\sigma}\left(\xi_{k}\right)}\left|\left(y-\xi_{k}\right) \cdot \nabla v_{j}\right|^{2}=0 .
$$

If $R$ is large and $\sigma$ is small, in $D=\cap_{k}\left(B_{R}\left(\xi_{k}\right) \backslash B_{\sigma}\left(\xi_{k}\right)\right) \cap\left(B_{R}(0) \backslash B_{\sigma}(0)\right)$,

$$
\begin{aligned}
& \lim _{j \rightarrow+\infty} \int_{D}\left|\xi_{k} \cdot \nabla v_{j}\right|^{2} \\
= & \lim _{j \rightarrow+\infty} \int_{D}\left|\left(y-\xi_{k}\right) \cdot \nabla v_{j}\right|^{2}-\left|y \cdot \nabla v_{j}\right|^{2}+2\left(y \cdot \nabla v_{j}\right)\left(\xi_{k} \cdot \nabla v_{j}\right) \\
\leq & \lim _{j \rightarrow+\infty} \int_{D}\left|\left(y-\xi_{k}\right) \cdot \nabla v_{j}\right|^{2}+10\left|y \cdot \nabla v_{j}\right|^{2}+\frac{1}{2}\left(\xi_{k} \cdot \nabla v_{j}\right)^{2} .
\end{aligned}
$$

So

$$
\lim _{j \rightarrow+\infty} \int_{D}\left|\xi_{k} \cdot \nabla v_{j}\right|^{2}=0 .
$$

Then a suitable covering using translations of $D$ gives the result. 
In the proof we have used the following fact. For any $\varepsilon>0$, there exists a constant $C$ such that, for all $j$ large,

$$
\sup _{\left\{\left|x^{\prime \prime}\right|>\varepsilon\right\}}\left|v_{j}\right| \leq C \text {. }
$$

In particular, $v_{j}$ are smooth outside the $\varepsilon$-neighborhood of $T$.

By Pacard's partial regularity result [15] for stationary solutions, $H^{n-m}\left(\mathcal{S}\left(v_{j}\right)\right)=0$. Since the projection $\pi$ from $\mathbb{R}^{n}$ to $T$ is a $1-$ Lipschitz map, direct calculation using the definition of Hausdorff measures gives

$$
H^{n-m}\left(\pi \mathcal{S}\left(v_{j}\right)\right)=0 \text {. }
$$

In other words, for $H^{n-m}$-a.a. $x^{\prime} \in T,\left(\left\{x^{\prime}\right\} \times \mathbb{R}^{m}\right) \cap \mathcal{S}\left(v_{j}\right)=\emptyset$. Thus for all $j$ large, $v_{j}\left(x^{\prime}, \cdot\right)$ are smooth functions in $\mathbb{R}^{m}$ for $H^{n-m}$-a.a. $x^{\prime} \in T$, and these points form an open set. (Note that the regular set of $v_{j}$ is open.)

Let

$$
f_{j}\left(x^{\prime}\right):=\int_{B_{1}^{\prime \prime}} \sum_{k=1}^{n-m}\left|\frac{\partial v_{j}}{\partial x_{k}}\left(x^{\prime}, x^{\prime \prime}\right)\right|^{2} d x^{\prime \prime} .
$$

Lemma 5.2 says $f_{j}$ converges to 0 in $L_{l o c}^{1}\left(\mathbb{R}^{n-m}\right)$. By the weak- $L^{1}$ estimate for the Hardy-Littlewood maximal function, for $H^{n-m}-$ a.a. $x^{\prime} \in T$,

$$
M f_{j}\left(x^{\prime}\right):=\sup _{0<r \leq 1 / 2} r^{m-n} \int_{B_{r}^{\prime}\left(x^{\prime}\right)} f_{j}\left(y^{\prime}\right) d y^{\prime} \rightarrow 0, \text { as } j \rightarrow+\infty .
$$

For any $\delta>0$, we can take an open set $E_{j} \subset B_{1}^{\prime}$ with

$$
H^{n-m}\left(E_{j}\right) \geq(1-\delta) H^{n-m}\left(B_{1}^{\prime}\right),
$$

such that for any $x^{\prime} \in E_{j}$, (5.4) holds and $v_{j}$ is smooth in a neighborhood of $\left\{x^{\prime}\right\} \times B_{1 / 2}^{\prime \prime}$.

Take an arbitrary sequence $x_{j}^{\prime} \in E_{j}$. Then for all $x^{\prime \prime} \in B_{1 / 2}^{\prime \prime}$,

$$
\lim _{\delta \rightarrow 0} \delta^{m-n} \int_{B_{\delta}\left(x_{j}^{\prime}, x^{\prime \prime}\right)} \frac{p-1}{2}\left|\nabla v_{j}\right|^{2}+\frac{p-1}{p+1}\left|v_{j}\right|^{p+1}=0 .
$$

On the other hand, by the definition of the blow up locus, there exists a $\delta_{j}>0$, which goes to 0 as $j \rightarrow+\infty$, such that

$$
\delta_{j}^{m-n} \int_{B_{\delta_{j}}\left(x_{j}^{\prime}, 0\right)} \frac{p-1}{2}\left|\nabla v_{j}\right|^{2}+\frac{p-1}{p+1}\left|v_{j}\right|^{p+1} \geq \frac{\varepsilon_{0}}{2} .
$$

These two imply the existence of an $r_{j} \in\left(0, \delta_{j}\right)$, such that

$$
\max _{x^{\prime \prime} \in B_{1 / 2}^{\prime \prime}} r_{j}^{m-n} \int_{B_{r_{j}}\left(x_{j}^{\prime}, x^{\prime \prime}\right)} \frac{p-1}{2}\left|\nabla v_{j}\right|^{2}+\frac{p-1}{p+1}\left|v_{j}\right|^{p+1}=c_{1} \varepsilon_{0},
$$


where we have chosen a fixed constant $c_{1} \in(0,1 / 2)$.

Assume this maxima is attained at $x_{j}^{\prime \prime}$, and denote $x_{j}=\left(x_{j}^{\prime}, x_{j}^{\prime \prime}\right)$. Define

$$
\bar{v}_{j}(x)=r_{j}^{\frac{2}{p-1}} v_{j}\left(x_{j}+r_{j} x\right) .
$$

By Lemma 2.4, for any $r \in\left(0, r_{j}^{-1} / 2\right)$,

$$
\int_{B_{r}(0)} \frac{p-1}{2}\left|\nabla \bar{v}_{j}\right|^{2}+\frac{p-1}{p+1}\left|\bar{v}_{j}\right|^{p+1} \leq C(M) r^{n-2 \frac{p+1}{p-1}} .
$$

Without loss of generality, assume that $\bar{v}_{j}$ converges weakly to $\bar{v}$ in $H_{\text {loc }}^{1}\left(\mathbb{R}^{n}\right) \cap L_{\text {loc }}^{p+1}\left(\mathbb{R}^{n}\right)$. By the compact Sobolev embedding, $\bar{v}_{j}$ converges to $\bar{v}$ in $\left.L_{l o c}^{q}\left(\mathbb{R}^{n}\right)\right)$ for any $q<p+1$. In particular, $\bar{v}$ is an $H^{1}$ weak solution of (1.1) in $\mathbb{R}^{n}$.

By (5.4) and Fatou lemma, for all $0<r \leq r_{j}^{-1} / 2$,

$$
\begin{aligned}
& r^{m-n} \int_{B_{r}(0)} \sum_{k=1}^{n-m}\left|\frac{\partial \bar{v}}{\partial x_{k}}\left(x^{\prime}, x^{\prime \prime}\right)\right|^{2} \\
\leq & \liminf _{j \rightarrow+\infty} r^{m-n} \int_{B_{r}(0)} \sum_{k=1}^{n-m}\left|\frac{\partial \bar{v}_{j}}{\partial x_{k}}\left(x^{\prime}, x^{\prime \prime}\right)\right|^{2} \\
= & \liminf _{j \rightarrow+\infty}\left(r r_{j}\right)^{m-n} \int_{B_{r r_{j}}\left(x_{j}\right)} \sum_{k=1}^{n-m}\left|\frac{\partial v_{j}}{\partial x_{k}}\left(x^{\prime}, x^{\prime \prime}\right)\right|^{2}=0 .
\end{aligned}
$$

Hence $\bar{v}\left(x^{\prime}, x^{\prime \prime}\right)=\bar{v}\left(x^{\prime \prime}\right)$. In (5.9), we can replace $B_{r}$ by the cylinder $B_{r / 2}^{\prime} \times B_{r / 2}^{\prime \prime}$, which then gives (noting that $m=2 \frac{p+1}{p-1}$ and $r$ can be arbitrarily large)

$$
\int_{\mathbb{R}^{m}}\left|\nabla^{\prime \prime} \bar{v}\left(x^{\prime \prime}\right)\right|^{2}+\left|v\left(x^{\prime \prime}\right)\right|^{p+1} d x^{\prime \prime} \leq C(M) .
$$

Because $\bar{v}$ is a solution to (1.1), with $p$ the critical exponent in $\mathbb{R}^{m}$, by $[19$, Lemma B.3] it is a smooth solution.

We can assume the measures

$$
\left(\left|\nabla \bar{v}_{j}\right|^{2}+\left|\bar{v}_{j}\right|^{p+1}\right) d x \rightarrow\left(|\nabla \bar{v}|^{2}+|\bar{v}|^{p+1}\right) d x+\tau .
$$

Here $\tau$ is a positive Radon measure.

Lemma 5.3. $\tau$ is translation invariant under $x^{\prime}$ direction.

Proof. For any $\varphi \in C_{0}^{\infty}\left(\mathbb{R}^{n}\right)$, define

$$
F_{j}(a)=\int_{\mathbb{R}^{n}}\left(\frac{1}{2}\left|\nabla \bar{v}_{j}\right|^{2}-\frac{1}{p+1}\left|\bar{v}_{j}\right|^{p+1}\right)(x+a) \varphi(x) d x .
$$


$F_{j}(a)$ are smooth functions of $a$. Then for $k=1, \cdots, n-m$,

$$
\frac{\partial F_{j}}{\partial a_{k}}=\int_{\mathbb{R}^{n}} \frac{\partial}{\partial x_{k}}\left(\frac{1}{2}\left|\nabla \bar{v}_{j}\right|^{2}-\frac{1}{p+1}\left|\bar{v}_{j}\right|^{p+1}\right)(x+a) \varphi(x) d x .
$$

Define $Y=\varphi(x) \frac{\partial}{\partial x_{k}}$, then $\operatorname{div} Y=\frac{\partial \varphi}{\partial x_{k}}$. By the stationary condition,

$$
\frac{\partial F_{j}}{\partial a_{k}}=-\sum_{l=1}^{n} \int_{\mathbb{R}^{n}} \frac{\partial \bar{v}_{j}}{\partial x_{k}}(x+a) \frac{\partial \bar{v}_{j}}{\partial x_{l}}(x+a) \frac{\partial \varphi}{\partial x_{l}}(x) d x .
$$

By (5.10), this goes to 0 uniformly on any compact set of $\mathbb{R}^{n}$. Since the measures

$$
\left(\frac{1}{2}\left|\nabla \bar{v}_{j}\right|^{2}-\frac{1}{p+1}\left|\bar{v}_{j}\right|^{p+1}\right) d x \rightarrow\left(\frac{1}{2}|\nabla \bar{v}|^{2}-\frac{1}{p+1}|\bar{v}|^{p+1}\right) d x+\frac{p-1}{4(p+1)} \tau,
$$

and $\left(\frac{1}{2}|\nabla \bar{v}|^{2}-\frac{1}{p+1}|\bar{v}|^{p+1}\right) d x$ is translation invariant in $x_{k}$ directions, $1 \leq k \leq n-m, \tau$ is also translation invariant in $x_{k}$ directions.

By the choice of $x_{j}$ (see (5.8)), we have

$$
c_{1} \varepsilon_{0}=\int_{B_{1}}\left|\nabla \bar{v}_{j}\right|^{2}+\left|\bar{v}_{j}\right|^{p+1}=\max _{x^{\prime \prime} \in B_{r_{j}^{\prime \prime} / 2}} \int_{B_{1}\left(0, x^{\prime \prime}\right)}\left|\nabla v_{j}\right|^{2}+\left|v_{j}\right|^{p+1} .
$$

Take two nonnegative functions $\varphi \in C_{0}^{\infty}\left(B_{2}^{\prime}\right), \psi \in C_{0}^{\infty}\left(B_{2}^{\prime \prime}\right)$, such that $\varphi \equiv 1$ in $B_{1}^{\prime}, \psi \equiv 1$ in $B_{1}^{\prime \prime}$. For $a \in B_{1}^{\prime} \times B_{1}^{\prime \prime}$, define

$$
F_{j}(a)=\int\left(\left|\nabla \bar{v}_{j}\right|^{2}+\left|\bar{v}_{j}\right|^{p+1}\right)(x+a) \varphi\left(x^{\prime}\right) \psi\left(x^{\prime \prime}\right) d x .
$$

Similar to the above discussion, $F_{j}$ are uniformly bounded in $C^{1}\left(B_{1}^{\prime} \times\right.$ $\left.B_{1}^{\prime \prime}\right)$, and it converges uniformly to

$$
\begin{aligned}
& \int\left(|\nabla \bar{v}|^{2}+|\bar{v}|^{p+1}\right) \varphi\left(x^{\prime}-a^{\prime}\right) \psi\left(x^{\prime \prime}-a^{\prime \prime}\right)+\int \varphi\left(x^{\prime}-a^{\prime}\right) \psi\left(x^{\prime \prime}-a^{\prime \prime}\right) d \tau \\
= & \int\left(|\nabla \bar{v}|^{2}+|\bar{v}|^{p+1}\right) \varphi\left(x^{\prime}\right) \psi\left(x^{\prime \prime}-a^{\prime \prime}\right)+\int \varphi\left(x^{\prime}\right) \psi\left(x^{\prime \prime}-a^{\prime \prime}\right) d \tau,
\end{aligned}
$$

which is independent of $a^{\prime}$. Thus for any $R>0$ fixed, if $j$ is large enough,

$$
\max _{x^{\prime} \in B_{2}^{\prime}, x^{\prime \prime} \in B_{R}^{\prime \prime}} \int_{B_{1}\left(x^{\prime}, x^{\prime \prime}\right)}\left|\nabla v_{j}\right|^{2}+\left|v_{j}\right|^{p+1} \leq 2 c_{1} \varepsilon_{0}<\varepsilon_{0} .
$$

By Theorem 2.2 and standard elliptic estimates, for any $k, \bar{v}_{j}$ are uniformly bounded in $C_{l o c}^{k}\left(B_{3 / 2}^{\prime}(0) \times B_{R-1}^{\prime \prime}(0)\right)$. Then we can take limit in (5.11) to get

$$
c_{1} \varepsilon_{0}=\int_{B_{1}}|\nabla \bar{v}|^{2}+|\bar{v}|^{p+1} .
$$


In particular, $\bar{v}$ is nontrivial.

In conclusion, after two rescalings from $u_{i}$, we construct a nontrivial smooth solution $\bar{v}$ to the equation

$$
-\Delta \bar{v}=|\bar{v}|^{\frac{4}{m-2}} \bar{v} \quad \text { in } \mathbb{R}^{m} .
$$

Moreover, $\bar{v}$ satisfies

$$
\int_{\mathbb{R}^{m}}\left|\nabla^{\prime \prime} \bar{v}\right|^{2}+|\bar{v}|^{\frac{2 m}{m-2}} \leq C(M) .
$$

This proves the last part of Theorem 1.2.

Note that positive solutions (to (1.1) in $\mathbb{R}^{m}$ ) have the least energy. By [2], up to a translation and scaling, for every $1 \leq i \leq N$, the positive solution has the form

$$
[m(m-2)]^{\frac{m-2}{4}}\left(1+\left|y^{\prime \prime}\right|^{2}\right)^{-\frac{m-2}{2}} .
$$

Since translations and scalings in $\mathbb{R}^{m}$ do not change the energy, there exists a constant $c(m)$ depending only on $m$, such that for any positive solution $\bar{v}$ of (1.1) on $\mathbb{R}^{m}$,

$$
\int_{\mathbb{R}^{m}} \frac{p-1}{2}|\nabla \bar{v}|^{2}+\frac{p-1}{p+1}|\bar{v}|^{p+1}=c(m) .
$$

For any $R>0$, by a rescaling and using the smooth convergence of $\bar{v}_{j}$ in $B_{R}$, we have

$$
\begin{aligned}
& \int_{B_{1}^{\prime \prime}}\left(\frac{p-1}{2}\left|\nabla v_{j}\left(x_{j}^{\prime}, x^{\prime \prime}\right)\right|^{2}+\frac{p-1}{p+1}\left|v_{j}\left(x_{j}^{\prime}, x^{\prime \prime}\right)\right|^{p+1}\right) d x^{\prime \prime} \\
\geq & \int_{B_{R r_{j}}^{\prime \prime}\left(x_{j}^{\prime \prime}\right)}\left(\frac{p-1}{2}\left|\nabla v_{j}\left(x_{j}^{\prime}, x^{\prime \prime}\right)\right|^{2}+\frac{p-1}{p+1}\left|v_{j}\left(x_{j}^{\prime}, x^{\prime \prime}\right)\right|^{p+1}\right) d x^{\prime \prime} \\
= & \int_{B_{R}^{\prime \prime}}\left(\frac{p-1}{2}\left|\nabla \bar{v}_{j}\left(0, x^{\prime \prime}\right)\right|^{2}+\frac{p-1}{p+1}\left|\bar{v}_{j}\left(0, x^{\prime \prime}\right)\right|^{p+1}\right) d x^{\prime \prime} \\
\rightarrow & \int_{B_{R}^{\prime \prime}}\left(\frac{p-1}{2}\left|\nabla \bar{v}\left(x^{\prime \prime}\right)\right|^{2}+\frac{p-1}{p+1}\left|\bar{v}_{j}\left(x^{\prime \prime}\right)\right|^{p+1}\right) d x^{\prime \prime} \\
\geq & c(m)-\sigma(R) .
\end{aligned}
$$

Here $\sigma(R)$ is defined by the following lemma.

Lemma 5.4. There exists a positive, continuous non-increasing function $\sigma(R)$ defined on $[0,+\infty)$ with

$$
\lim _{R \rightarrow+\infty} \sigma(R)=0
$$


such that for any solution $v$ of (1.1) on $\mathbb{R}^{m}$, satisfying (5.12),

$$
\int_{B_{R}^{\prime \prime}} \frac{p-1}{2}\left|\nabla^{\prime \prime} v\right|^{2}+\frac{p-1}{p+1}|v|^{\frac{2 m}{m-2}} \geq c(m)-\sigma(R) .
$$

Proof. Arguing by contradiction, we can assume that there exist a constant $\sigma>0$, a sequence of solutions $v_{j}$ satisfying all of the assumptions, and $R_{j} \rightarrow+\infty$ such that

$$
\int_{B_{R_{j}}^{\prime \prime}} \frac{p-1}{2}\left|\nabla^{\prime \prime} v_{j}\right|^{2}+\frac{p-1}{p+1}\left|v_{j}\right|^{\frac{2 m}{m-2}}<c(m)-\sigma .
$$

With the above uniform bound we can assume that $v_{j}$ converges weakly to $v$ in $H_{l o c}^{1}\left(\mathbb{R}^{m}\right) \cap L_{l o c}^{2 m /(m+2)}\left(\mathbb{R}^{m}\right)$. If $v$ is nonzero, by Fatou lemma we get

$$
\int_{\mathbb{R}^{m}} \frac{p-1}{2}\left|\nabla^{\prime \prime} v\right|^{2}+\frac{p-1}{p+1}|v|^{\frac{2 m}{m-2}} \leq c(m)-\sigma .
$$

This is a contradiction since the lowest energy is exactly $c(m)$.

If $v=0$, by (5.12) and Struwe's global compactness theorem [19, Theorem 3.1], there must exists an blow up point $x_{0} \in B_{2}^{\prime \prime}$ such that (at least) one bubble concentrates at $x_{0}$. More precisely, there exists $x_{j}^{\prime \prime} \rightarrow x_{0}$ and $r_{j} \rightarrow 0$ such that

$$
r_{j}^{\frac{m-2}{2}} v_{j}\left(x_{j}^{\prime \prime}+r_{j} x^{\prime \prime}\right)
$$

converges to a nontrivial solution of (1.1) weakly in $H_{l o c}^{1}\left(\mathbb{R}^{m}\right) \cap L_{l o c}^{2 m /(m+2)}\left(\mathbb{R}^{m}\right)$. This bubble carries energy at least $c(m)$, which is concentrated in a small ball around $x_{0}$. Thus we get a contradiction once again.

Since (5.14) holds for any $x^{\prime} \in E_{j}$, by noting (5.5), we get

$$
\begin{aligned}
& \liminf _{j \rightarrow+\infty} \int_{B_{1}^{\prime}} \int_{B_{1}^{\prime \prime}}\left(\frac{p-1}{2}\left|\nabla v_{j}\left(x^{\prime}, x^{\prime \prime}\right)\right|^{2}+\frac{p-1}{p+1}\left|v_{j}\left(x^{\prime}, x^{\prime \prime}\right)\right|^{p+1}\right) d x^{\prime \prime} d x^{\prime} \\
\geq & \liminf _{j \rightarrow+\infty} \int_{E_{j}} \int_{B_{1}^{\prime \prime}}\left(\frac{p-1}{2}\left|\nabla v_{j}\left(x^{\prime}, x^{\prime \prime}\right)\right|^{2}+\frac{p-1}{p+1}\left|v_{j}\left(x^{\prime}, x^{\prime \prime}\right)\right|^{p+1}\right) d x^{\prime \prime} d x^{\prime} \\
\geq & (c(m)-\sigma(R))(1-\delta) H^{n-m}\left(B_{1}^{\prime}\right) .
\end{aligned}
$$

After letting $R \rightarrow+\infty$ and $\delta \rightarrow 0$, and noting (5.1), we obtain

Corollary 5.5. For $H^{n-m}-$ a.a. $x \in \Sigma, \Theta(x) \geq c(m)$. 


\section{Quantization of Density Function}

In the previous section we have constructed a sequence of $v_{j}$ such that (here we use the same notations as in the previous section)

$$
\left(\frac{p-1}{2}\left|\nabla v_{j}\right|^{2}+\frac{p-1}{p+1}\left|v_{j}\right|^{p+1}\right) d x \rightarrow \Theta(0) H^{n-m}{\left\lfloor\mathbb{R}^{n-m}\right.} .
$$

In this section we prove the quantization of $\Theta(0)$, under the following assumption

$$
\Delta^{\prime} v_{j} \rightarrow 0, \quad \text { in } L_{l o c}^{\frac{p+1}{p}}\left(\mathbb{R}^{n}\right)
$$

Note that since $v_{j}$ are uniformly bounded in $L_{l o c}^{p+1}\left(\mathbb{R}^{n}\right)$, by standard interior $W^{2, \frac{p+1}{p}}$ estimates, $D^{2} v_{j}$ are uniformly bounded in $L_{l o c}^{\frac{p+1}{p}}\left(\mathbb{R}^{n}\right)$. In view of Lemma 5.2, it is natural to conjecture that (6.2) holds.

Theorem 6.1. There exists at most $N$ solutions of (1.1) in $\mathbb{R}^{m}, w_{i}$, $1 \leq i \leq N$, with

$$
\int_{\mathbb{R}^{m}} \frac{p-1}{2}\left|\nabla w_{i}\right|^{2}+\frac{p-1}{p+1}\left|w_{i}\right|^{p+1}<+\infty
$$

such that

$$
\Theta(0)=\sum_{i=1}^{N} \int_{\mathbb{R}^{m}} \frac{p-1}{2}\left|\nabla w_{i}\right|^{2}+\frac{p-1}{p+1}\left|w_{i}\right|^{p+1} .
$$

Here

$$
N \leq \frac{C(M)}{c(m)}
$$

Before proving this theorem, we first show that the problem can be reduced to a slice.

Lemma 6.2. For a.a. $x^{\prime} \in B_{1}^{\prime}$, on $B_{1}^{\prime \prime}$,

$$
\left(\frac{p-1}{2}\left|\nabla v_{j}\left(x^{\prime}, x^{\prime \prime}\right)\right|^{2}+\frac{p-1}{p+1}\left|v_{j}\left(x^{\prime}, x^{\prime \prime}\right)\right|^{p+1}\right) d x^{\prime \prime} \rightarrow \Theta(0) \delta_{0},
$$

where $\delta_{0}$ is the Dirac measure supported at the origin $0 \in \mathbb{R}^{m}$.

Proof. Fix a $\varphi \in C_{0}^{\infty}\left(B_{1}^{\prime \prime}\right)$ such that $0 \leq \varphi \leq 1, \varphi \equiv 1$ in $B_{1 / 2}^{\prime \prime}$. For any smooth vector field $X \in C_{0}^{\infty}\left(B_{1}^{\prime}, \mathbb{R}^{n-m}\right)$, substitute $\varphi X$ into the stationary condition (1.2). This leads to

$$
\begin{aligned}
0= & \int_{B_{1}^{\prime}} \int_{B_{1}^{\prime \prime}}\left(\frac{1}{2}\left|\nabla v_{j}\right|^{2}-\frac{1}{p+1}\left|v_{j}\right|^{p+1}\right) \varphi \operatorname{div} X d x^{\prime \prime} d x^{\prime} \\
& -\int_{B_{1}^{\prime}} \int_{B_{1}^{\prime \prime}}\left[\varphi D X\left(\nabla^{\prime} v_{j}, \nabla^{\prime} v_{j}\right)+\left(\nabla^{\prime \prime} \varphi \cdot \nabla^{\prime \prime} v_{j}\right)\left(X \cdot \nabla^{\prime} v_{j}\right)\right] d x^{\prime \prime} d x^{\prime}
\end{aligned}
$$


By using the equation (1.1) and integrating by parts, we have

$$
\begin{aligned}
& \int_{B_{1}^{\prime}} \int_{B_{1}^{\prime \prime}}\left|v_{j}\right|^{p+1} \varphi \operatorname{div} X d x^{\prime \prime} d x^{\prime} \\
= & -\int_{B_{1}^{\prime}} \int_{B_{1}^{\prime \prime}} \Delta v_{j} v_{j} \varphi \operatorname{div} X d x^{\prime \prime} d x^{\prime} \\
= & \int_{B_{1}^{\prime}} \int_{B_{1}^{\prime \prime}}\left|\nabla v_{j}\right|^{2} \varphi \operatorname{div} X d x^{\prime \prime} d x^{\prime} \\
& +\int_{B_{1}^{\prime}} \int_{B_{1}^{\prime \prime}}\left[v_{j}\left(\nabla^{\prime} v_{j} \cdot \nabla^{\prime} \operatorname{div} X\right) \varphi+v_{j}\left(\nabla^{\prime \prime} v_{j} \cdot \nabla^{\prime \prime} \varphi\right) \operatorname{div} X\right] d x^{\prime \prime} d x^{\prime} .
\end{aligned}
$$

Integrating by parts once again,

$$
\begin{aligned}
& \int_{B_{1}^{\prime}} \int_{B_{1}^{\prime \prime}} v_{j}\left(\nabla^{\prime} v_{j} \cdot \nabla^{\prime} \operatorname{div} X\right) \varphi d x^{\prime \prime} d x^{\prime} \\
= & -\int_{B_{1}^{\prime}} \int_{B_{1}^{\prime \prime}}\left(\left|\nabla^{\prime} v_{j}\right|^{2}+\Delta^{\prime} v_{j} v_{j}\right) \operatorname{div} X \varphi d x^{\prime \prime} d x^{\prime} .
\end{aligned}
$$

By Lemma 5.2, (6.2) and the uniform $L^{p+1}\left(B_{1}^{\prime} \times B_{1}^{\prime \prime}\right)$ bound on $v_{j}$,

$$
\begin{gathered}
\lim _{j \rightarrow \infty} \int_{B_{1}^{\prime}} \int_{B_{1}^{\prime \prime}}\left(\left|\nabla^{\prime} v_{j}\right|^{2}+\left|\Delta^{\prime} v_{j} v_{j}\right|\right) d x^{\prime \prime} d x^{\prime}=0, \\
\lim _{j \rightarrow \infty} \int_{B_{1}^{\prime}} \int_{B_{1}^{\prime \prime}} \sum_{k, l=1}^{n-m}\left|\frac{\partial v_{j}}{\partial x_{k}}\right|\left|\frac{\partial v_{j}}{\partial x_{l}}\right| d x^{\prime \prime} d x^{\prime}=0 .
\end{gathered}
$$

By Lemma 5.2 and the uniform $L^{2}\left(B_{1}^{\prime} \times B_{1}^{\prime \prime}\right)$ bound on $\nabla^{\prime \prime} v_{j}$ and Cauchy inequality,

$$
\lim _{j \rightarrow \infty} \int_{B_{1}^{\prime}} \int_{B_{1}^{\prime \prime}}\left|\left(\nabla^{\prime \prime} \varphi \cdot \nabla^{\prime \prime} v_{j}\right) \nabla^{\prime} v_{j}\right| d x^{\prime \prime} d x^{\prime}=0
$$

Because $v_{j} \rightarrow 0$ in $L^{2}\left(B_{1}^{\prime} \times B_{1}^{\prime \prime}\right.$ ) (note that $v_{j}$ converges uniformly to 0 on any compact set in $\left.B_{1}^{\prime} \times\left(B_{1}^{\prime \prime} \backslash\{0\}\right)\right)$, combining this with the uniform $L^{2}\left(B_{1}^{\prime} \times B_{1}^{\prime \prime}\right)$ bound on $\nabla^{\prime \prime} v_{j}$ and Cauchy inequality, we get

$$
\lim _{j \rightarrow \infty} \int_{B_{1}^{\prime}} \int_{B_{1}^{\prime \prime}}\left|v_{j}\left(\nabla^{\prime \prime} v_{j} \cdot \nabla^{\prime \prime} \varphi\right)\right| d x^{\prime \prime} d x^{\prime}=0 .
$$

Substituting (6.5)-(6.9) into (6.4), we obtain

$$
\int_{B_{1}^{\prime}}\left[\int_{B_{1}^{\prime \prime}}\left(\frac{1}{2}-\frac{1}{p+1}\right)\left|\nabla v_{j}\right|^{2} \varphi d x^{\prime \prime}\right] \operatorname{div} X d x^{\prime}=\int_{B_{1}^{\prime}} f_{j} \cdot X+A_{j} \cdot D X d x^{\prime}
$$


where $f_{j} \in L^{1}\left(B_{1}^{\prime}, \mathbb{R}^{n-m}\right)$ and $A_{j} \in L^{1}\left(B_{1}^{\prime}, \mathbb{R}^{n-m} \otimes \mathbb{R}^{n-m}\right)$ (i.e. $A_{j}$ are matrix valued), satisfying

$$
\lim _{j \rightarrow \infty} \int_{B_{1}}\left|f_{j}\right|+\left|A_{j}\right| d x^{\prime}=0 .
$$

By (6.1) and Lemma 2.6,

$$
\left[\int_{B_{1}^{\prime \prime}}\left(\frac{1}{2}-\frac{1}{p+1}\right)\left|\nabla v_{j}\right|^{2} \varphi d x^{\prime \prime}\right] \rightarrow \frac{\Theta(0)}{p+3}, \quad \text { weakly in } L^{1}\left(B_{1}^{\prime}\right) .
$$

Then we can apply Allard's strong constancy lemma (cf. [1, Section 1]) to conclude that

$$
\int_{B_{1}^{\prime \prime}}\left(\frac{1}{2}-\frac{1}{p+1}\right)\left|\nabla v_{j}\right|^{2} \varphi d x^{\prime \prime} \rightarrow \frac{\Theta(0)}{p+3}, \quad \text { in } L^{1}\left(B_{1}^{\prime}\right) .
$$

We can also substitute (6.5) into (6.4) to eliminate $\left|\nabla v_{j}\right|^{2}$. This leads to the strong convergence of

$$
\int_{B_{1}^{\prime \prime}}\left(\frac{1}{2}-\frac{1}{p+1}\right)\left|v_{j}\right|^{p+1} \varphi d x^{\prime \prime}
$$

in $L^{1}\left(B_{1}^{\prime}\right)$.

Now we have proved that

$$
\int_{B_{1}^{\prime \prime}}\left(\frac{p-1}{2}\left|\nabla v_{j}\right|^{2}+\frac{p-1}{p+1}\left|v_{j}\right|^{p+1}\right) \varphi d x^{\prime \prime} \rightarrow \Theta(0)
$$

in $L^{1}\left(B_{1}^{\prime}\right)$. Since $v_{j}$ converges to 0 in $C^{1}\left(B_{1}^{\prime} \times\left(B_{1}^{\prime \prime} \backslash B_{1 / 3}^{\prime \prime}\right)\right.$ and $1-\varphi \equiv 0$ in $B_{1 / 2}^{\prime \prime}$,

$$
\lim _{j \rightarrow 0} \int_{B_{1}^{\prime}} \int_{B_{1}^{\prime \prime}}\left(\frac{p-1}{2}\left|\nabla v_{j}\right|^{2}+\frac{p-1}{p+1}\left|v_{j}\right|^{p+1}\right)(1-\varphi) d x^{\prime \prime} d x^{\prime}=0 .
$$

Thus

$$
\int_{B_{1}^{\prime \prime}}\left(\frac{p-1}{2}\left|\nabla v_{j}\left(x^{\prime}, x^{\prime \prime}\right)\right|^{2}+\frac{p-1}{p+1}\left|v_{j}\left(x^{\prime}, x^{\prime \prime}\right)\right|^{p+1}\right) d x^{\prime \prime} \rightarrow \Theta(0)
$$

in $L^{1}\left(B_{1}^{\prime}\right)$.

After passing to a subsequence of $j$, we can assume that for a.a. $x^{\prime} \in B_{1}^{\prime}$,

$$
\int_{B_{1}^{\prime \prime}}\left(\frac{p-1}{2}\left|\nabla v_{j}\left(x^{\prime}, x^{\prime \prime}\right)\right|^{2}+\frac{p-1}{p+1}\left|v_{j}\left(x^{\prime}, x^{\prime \prime}\right)\right|^{p+1}\right) d x^{\prime \prime} \rightarrow \Theta(0) .
$$


Then by noting that

$$
\left(\frac{p-1}{2}\left|\nabla v_{j}\left(x^{\prime}, x^{\prime \prime}\right)\right|^{2}+\frac{p-1}{p+1}\left|v_{j}\left(x^{\prime}, x^{\prime \prime}\right)\right|^{p+1}\right) \rightarrow 0,
$$

uniformly on any compact set of $B_{1}^{\prime \prime} \backslash\{0\}$, we must have

$$
\left(\frac{p-1}{2}\left|\nabla v_{j}\left(x^{\prime}, x^{\prime \prime}\right)\right|^{2}+\frac{p-1}{p+1}\left|v_{j}\left(x^{\prime}, x^{\prime \prime}\right)\right|^{p+1}\right) d x^{\prime \prime} \rightarrow \Theta(0) \delta_{0},
$$

weakly as measures.

Proof of Theorem 6.1. By (6.2) and the previous lemma, for each $j$, there exists a closed set $E_{j} \subset B_{1}^{\prime}$ with $\lim _{j \rightarrow+\infty} H^{n-m}\left(E_{j}\right)=0$, such that for any $x_{j}^{\prime} \in B_{1}^{\prime} \backslash E_{j}$, (6.3) holds and

$$
\lim _{j \rightarrow \infty} \int_{B_{1}^{\prime \prime}}\left|\Delta^{\prime \prime} v_{j}\left(x_{j}^{\prime}, x^{\prime \prime}\right)\right|^{\frac{2 m}{m+2}} d x^{\prime \prime}=0 .
$$

Moreover, as in the previous section, we can also restrict $E_{j}$ further so that $v_{j}$ is smooth in a neighborhood of $E_{j} \times B_{1}^{\prime \prime}$.

Let $x_{j}^{\prime} \in B_{1}^{\prime} \backslash E_{j}$ be an arbitrary sequence, and

$$
\tilde{v}_{j}\left(x^{\prime}\right)=v_{j}\left(x_{j}^{\prime}, x^{\prime \prime}\right) \text {. }
$$

Thanks to (6.3), $\tilde{v}_{j}$ are uniformly bounded in $H^{1}\left(B_{1}^{\prime \prime}\right) \cap L^{\frac{2 m}{m-2}}\left(B_{1}^{\prime \prime}\right)$.

Since $v_{j}$ is smooth in a neighborhood of $\left\{x_{j}^{\prime}\right\} \times B_{1}^{\prime \prime}$,

$$
\Delta^{\prime \prime} \tilde{v}_{j}+\left|\tilde{v}_{j}\right|^{p-1} \tilde{v}_{j}=-\Delta^{\prime} \tilde{v}_{j}
$$

Thus by (6.10) and the Sobolev embedding theorem in dimension $m$, for any $\varphi \in H_{1}\left(B_{1}^{\prime \prime}\right)$,

$$
\begin{aligned}
\int_{B_{1}^{\prime \prime}}\left(\Delta^{\prime \prime} \tilde{v}_{j}+\left|\tilde{v}_{j}\right|^{p-1} \tilde{v}_{j}\right) \varphi & =\int_{B_{1}^{\prime \prime}}-\Delta^{\prime} \tilde{v}_{j} \varphi \\
& \leq\left(\int_{B_{1}^{\prime \prime}}\left|\Delta^{\prime \prime} v_{j}\left(x_{j}^{\prime}, x^{\prime \prime}\right)\right|^{\frac{2 m}{m+2}}\right)^{\frac{m+2}{2 m}}\left(\int_{B_{1}^{\prime \prime}}|\varphi|^{\frac{2 m}{m-2}}\right)^{\frac{m-2}{2 m}} \\
& =o_{j}\left(\|\varphi\|_{H^{1}\left(B_{1}\right)}\right) .
\end{aligned}
$$

Hence $\tilde{v}_{j}$ is a Palais-Smale sequence.

Then by [19, Theorem 3.1] (see also [6, Section 3.1]), we get $N$ functions $w_{i}\left(x^{\prime \prime}\right), 1 \leq i \leq N$, with

$$
-\Delta^{\prime \prime} w_{i}=\left|w_{i}\right|^{\frac{4}{m-2}} w_{i}, \quad \int_{\mathbb{R}^{m}}\left|\nabla^{\prime \prime} w_{i}\right|^{2}+\left|w_{i}\right|^{\frac{2 m}{m-2}}<+\infty,
$$


and $N$ points $x_{i, j}$ and radius $R_{i, j}>0,1 \leq i \leq N$, such that

$$
\left\|\tilde{v}_{j}-\sum_{i=1}^{N} R_{i, j}^{\frac{m-2}{2}} w_{i}\left(R_{i, j}\left(x-x_{i, j}\right)\right)\right\|_{H^{1}\left(B_{1}^{\prime \prime}\right)} \rightarrow 0 .
$$

Their $L^{\frac{2 m}{m-2}}\left(B_{1}^{\prime \prime}\right)$ norm also converge to 0 .

By Lemma 3.9 and (6.3),

$$
\lim _{j \rightarrow+\infty} \int_{B_{1}^{\prime \prime}} \frac{p-1}{2}\left|\nabla \tilde{v}_{j}\right|^{2}+\frac{p-1}{p+1}\left|\tilde{v}_{j}\right|^{p+1}=\Theta(0) \leq C(M) .
$$

There also exists a constant $c_{1}(m)$ depending only on $m$ such that (see Lemma 5.4)

$$
\int_{\mathbb{R}^{m}} \frac{p-1}{2}\left|\nabla w_{i}\right|^{2}+\frac{p-1}{p+1}\left|w_{i}\right|^{p+1} \geq c(m) .
$$

Note that this energy is invariant under scaling and translations in $\mathbb{R}^{m}$. Combining (6.11)-(6.13) we get the upper bound for $N$.

If the sequence of solutions are positive, then $w_{i} \geq 0$ for every $1 \leq$ $i \leq N$ (for a proof see [6, Section 3.2]). By [2], up to a translation and scaling, for every $1 \leq i \leq N$,

$$
w_{i}\left(y^{\prime \prime}\right)=[m(m-2)]^{\frac{m-2}{4}}\left(1+\left|y^{\prime \prime}\right|^{2}\right)^{-\frac{m-2}{2}} .
$$

Note that the translation and scaling do not change the energy of $w_{i}$, so

$$
\begin{aligned}
& \int_{\mathbb{R}^{m}} \frac{p-1}{2}\left|\nabla w_{i}\right|^{2}+\frac{p-1}{p+1}\left|w_{i}\right|^{p+1} \\
= & 4 \alpha(m)(m-1)[m(m-2)]^{\frac{m-2}{2}} \int_{0}^{\infty}\left(1+r^{2}\right)^{-m} r^{m-1} d r \\
= & c(m) .
\end{aligned}
$$

Together with (6.4) and (6.11), this gives

Corollary 6.3. In the case of positive solutions, there exists a constant $c(m)$ depending only on $m$ such that, for $H^{n-m}-a . a . x \in \Sigma, \Theta(x) / c(m)$ is an integer.

\section{Stationary Property of Blow up Locus}

In this section we prove that the stationary property of the limit function $u$ is equivalent to that of the blow-up locus. 
For any smooth vector field $Y$ with compact support, the stationary condition for $u_{i}$ says

$$
\int\left(\frac{1}{2}\left|\nabla u_{i}\right|^{2}-\frac{1}{p+1}\left|u_{i}\right|^{p+1}\right) \operatorname{div} Y-D Y\left(\nabla u_{i}, \nabla u_{i}\right)=0 .
$$

Let the Radon measure

$$
\gamma:=\frac{m(m-2)}{4(m-1)} \nu
$$

Then we have the weak convergence

$$
\left|\nabla u_{i}\right|^{2} d x \rightarrow|\nabla u|^{2} d x+\gamma
$$

Let

$$
\tau_{\alpha \beta}^{i}:=\left|\nabla u_{i}\right|^{-2} \frac{\partial u_{i}}{\partial x_{\alpha}} \frac{\partial u_{i}}{\partial x_{\beta}}
$$

if $\left|\nabla u_{i}\right| \neq 0$ (if $\left|\nabla u_{i}\right|=0$, we simply take $\left[\tau_{\alpha \beta}^{i}\right]=0$ ). We can assume the matrix-valued measures

$$
\tau_{\alpha \beta}^{i}\left|\nabla u_{i}\right|^{2} d x \rightarrow \frac{\partial u}{\partial x_{\alpha}} \frac{\partial u}{\partial x_{\beta}} d x+\tau_{\alpha \beta} d \nu .
$$

Here $\left[\tau_{\alpha \beta}\right]$ is symmetric, non-negative definite, measurable with respect to $\gamma$, and $\sum_{\alpha=1}^{n} \tau_{\alpha \alpha}=1 \gamma$-a.e.. By passing to the limit in the stationary condition for $u_{i}$, we get

$$
\begin{aligned}
0= & \int\left(\frac{1}{2}|\nabla u|^{2}-\frac{1}{p+1}|u|^{p+1}\right) \operatorname{div} Y-D Y(\nabla u, \nabla u) \\
& +2 \int\left(\frac{1}{m} \operatorname{div} Y-\sum_{\alpha, \beta=1}^{n} D_{\alpha} Y^{\beta} \tau_{\alpha \beta}\right) d \gamma
\end{aligned}
$$

For any $1 \leq \alpha, \beta \leq n, \tau_{\alpha \beta}$ is approximate continuous in the following sense: given an $\varepsilon>0$, for $\gamma-$ a.a. $x_{0}$,

$$
\lim _{r \rightarrow 0} \frac{\gamma\left(\left\{\left|\tau_{\alpha \beta}(x)-\tau_{\alpha \beta}\left(x_{0}\right)\right|>\varepsilon\right\} \cap B_{r}\left(x_{0}\right)\right)}{\gamma\left(B_{r}\left(x_{0}\right)\right)}=0 .
$$

Assume that $0 \in \Sigma=\operatorname{spt}(\gamma)$ satisfies this condition for all $1 \leq \alpha, \beta \leq n$, and $\mu_{0}=\Theta(0) H^{n-m}{\left\lfloor\mathbb{R}^{n-m}\right.}$ is defined as in Section 5. Then as $\lambda_{j} \rightarrow 0$,

$$
\begin{aligned}
\lim _{j \rightarrow+\infty} L_{\sharp}^{\lambda_{j}}\left[\tau_{\alpha \beta}(x) d \gamma\right] & =\frac{m(m-2)}{4(m-1)} \tau_{\alpha \beta}(0) d \mu_{0} \\
& =\frac{m(m-2)}{4(m-1)} \Theta(0) \tau_{\alpha \beta}(0) H^{n-m}{\left\lfloor\mathbb{R}^{n-m}\right.}
\end{aligned}
$$


For the sequence $v_{j}$ constructed in Section 5, similar to the discussion above,

$$
\frac{\partial v_{j}}{\partial x_{\alpha}} \frac{\partial v_{j}}{\partial x_{\beta}} d x \rightarrow \frac{m(m-2)}{4(m-1)} \tau_{\alpha \beta}(0) d \mu_{0} .
$$

The stationary condition for $v_{j}$ can be written as

$$
\int\left(\frac{1}{2}\left|\nabla v_{j}\right|^{2}-\frac{1}{p+1}\left|v_{j}\right|^{p+1}\right) \operatorname{div} Y-\sum_{\alpha, \beta=1}^{n} D_{\alpha} Y^{\beta} \frac{\partial v_{j}}{\partial x_{\alpha}} \frac{\partial v_{j}}{\partial x_{\beta}}=0 .
$$

Passing to the limit, by Lemma 2.6, we get

$$
\int\left(\frac{1}{2}-\frac{m-2}{2 m}\right) \operatorname{div} Y d \mu_{0}-\sum_{\alpha, \beta=1}^{n} D_{\alpha} Y^{\beta} \tau_{\alpha \beta}(0) d \mu_{0}=0 .
$$

Since $\mu_{0}=\Theta(0) H^{n-m}{\left\lfloor\mathbb{R}^{n-m}\right.}$, this can be written as

$$
\int_{\mathbb{R}^{n-m}} \sum_{\alpha, \beta=1}^{n}\left(\frac{1}{m} \delta_{\alpha \beta}-\tau_{\alpha \beta}(0)\right) D_{\alpha} Y^{\beta} d H^{n-m}=0 .
$$

Here $\delta_{\alpha \beta}$ is the Kronecker symbol.

First, for any $n-m+1 \leq k \leq n$ and $\eta \in C_{0}^{\infty}\left(\mathbb{R}^{n}\right)$, by choosing $Y^{\beta}=\eta \delta_{k \beta}$, we see

$$
\tau_{\alpha \beta}(0)=\frac{1}{m} \delta_{\alpha \beta}, \quad \text { if } \alpha \text { or } \beta \geq n-m+1 .
$$

Let $\left[A_{\alpha \beta}\right]=\left[\tau_{\alpha \beta(0)}\right]_{1 \leq \alpha, \beta \leq n-m}$. It is symmetric, nonnegative definite, with

$$
\sum_{\alpha=1}^{n-m} A_{\alpha \alpha}=\sum_{\alpha=1}^{n} \tau_{\alpha \alpha}(0)-\sum_{\alpha=n-m+1}^{n} \tau_{\alpha \alpha}(0)=0 .
$$

Thus $A=0$. This implies

$$
\left[\tau_{\alpha \beta}\right]=\frac{1}{m}(I d-S),
$$

where $I d$ is the identity operator on $\mathbb{R}^{n}$ and $S$ is the projection operator onto $\mathbb{R}^{n-m}$, i.e. the tangent plane of $\Sigma$ at 0 . (Note that this tangent plane exists uniquely $H^{n-m}$-a.e. on $\Sigma$, because $\Sigma$ is $(n-m)-$ rectifiable.)

Substituting this into (7.1), we get

$$
\begin{aligned}
0= & \int\left(\frac{1}{2}|\nabla u|^{2}-\frac{1}{p+1}|u|^{p+1}\right) \operatorname{div} Y-D Y(\nabla u, \nabla u) \\
& +\frac{2}{m} \int D Y \cdot S d \gamma
\end{aligned}
$$


Let $G^{n-m}$ be the $(n-m)$-dimensional Grassmanian space of $\mathbb{R}^{n}$, i.e. the space of (unoriented) $(n-m)$-dimensional subspace in $\mathbb{R}^{n}$. Define the varifold $V_{\Sigma}$ associated to $\Sigma$ as

$$
<V_{\Sigma}, \Phi>=\int_{\Sigma} \Phi(x, S(x)) d \gamma(x),
$$

for any $\Phi \in C_{0}^{0}\left(B_{1} \times G^{n-m}\right)$. Here $S(x)$ is the weak tangent plane of $\Sigma$ at $x$, which exists uniquely $H^{n-m}$-a.e. in $\Sigma$. We say $V_{\Sigma}$ is stationary if for any smooth vector field $Y$ with compact support (see [10, Section 6.2] for more discussions),

$$
\int_{\Sigma} D Y \cdot S(x) d \gamma(x)=0 .
$$

By (7.2) we get

Theorem 7.1. $u$ is a stationary solution if and only if $V_{\Sigma}$ is stationary.

\section{REFERENCES}

[1] W. Allard, An integrality theorem and a regularity theorem for surfaces whose first variation with respect to a parametric elliptic integrand is controlled, Proc. Symp. Pure Math. 44 (1986),1-28.

[2] L. Caffarelli, B. Gidas and J. Spruck, Asymptotic symmetry and local behavior of semilinear elliptic equations with critical Sobolev growth, Comm. Pure Appl. Math. 42 (1989), 271-297.

[3] E.N. Dancer, Infinitely many turning points for some supercritical problems. Ann. Mat. Pura Appl. (4) 178 (2000), 225-233.

[4] M. del Pino, M. Musso and F. Pacard, Bubbling along boundary geodesics near the second critical exponents, J. Eur. Math. Soc. $12(2010)$, no. $6,1553-1605$.

[5] W. Ding, J. Li and W. Li, Nonstationary weak limit of a stationary harmonic map sequence. Comm. Pure Appl. Math. 56 (2003), no. 2, $270-277$.

[6] O. Druet, E. Hebey and F. Robert, Blow-up theory for elliptic PDEs in Riemannian geometry. Mathematical Notes, 45. Princeton University Press, Princeton, NJ, 2004. viii+218 pp.

[7] F. Mahmoudi, Energy quantization for Yamabe's problem in conformal dimension. Electron. J. Differential Equations 2006, No. 71, 17 pp.

[8] Z. Guo and J. Li, The blow up locus of semilinear elliptic equations with supercritical exponents. Calc. Var. Partial Differential Equations 15 (2002), no. 2, 133-153.

[9] F.-H. Lin, Gradient estimates and blow-up analysis for stationary harmonic maps. Ann. of Math. (2) 149 (1999), no. 3, 785-829.

[10] F.-H. Lin and X. Yang, Geometric measure theory: an introduction. Advanced Mathematics (Beijing/Boston), Science Press, Beijing; International Press, Boston, 2002. 
[11] F.-H. Lin and T. Rivière, Energy quantization for harmonic maps. Duke Math. J. 111 (2002), no. 1, 177-193.

[12] J. Li and G. Tian, A blow-up formula for stationary harmonic maps. Internat. Math. Res. Notices 1998, no. 14, 735-755.

[13] J.M. Marstrand, The $(\Phi, s)$-regular subsets of $n$-spaces, Trans. Amer. Math. Soc. 113(1964), 369-392.

[14] F. Pacard, Partial regularity for weak solutions of a nonlinear elliptic equation. Manuscripta Mathematica 79 (1993), no. 1, 161-172.

[15] F. Pacard, Convergence and partial regularity for weak solutions of some nonlinear elliptic equation: the supercritical case. Annales de l'Institut Henri Poincaré. Analyse non linéaire vol.11 (1994), no. 5, 537-551.

[16] D. Preiss, Geometry of measures in $R^{n}$ : distribution, rectifiability and density, Annals of Math. 125 (1987), 537-643.

[17] T. Riviére, Interpolation spaces and energy quantization for Yang-Mills fields. Comm. Anal. Geom. 10 (2002), no. 4, 683-708.

[18] L. Simon, Lectures on geometric measure theory. Proceedings of the Centre for Mathematical Analysis, Australian National University, 3. Australian National University, Centre for Mathematical Analysis, Canberra, 1983.

[19] M. Struwe, Variational methods. Applications to nonlinear partial differential equations and Hamiltonian systems. Fourth edition. SpringerVerlag, Berlin, 2008.

[20] L. Tartar, Imbedding theorems of Sobolev spaces into Lorentz spaces, Boll. U.M.I. 1 (1998), 479-500.

[21] G. Tian, Gauge theory and calibrated geometry. I. Ann. of Math. (2) 151 (2000), no. 1, 193-268.

[22] K. Wang, Partial regularity of stable solutions to the supercritical equations and its applications, Nonlinear Anal. 75 (2012), no. 13, 5238-5260.

K. Wang- Wuhan Institute of Physics and Mathematics, The Chinese Academy of Sciences, Wuhan 430071, China.

E-mail address: wangkelei@wipm.ac.cn

J. Wei - Department Of Mathematics, University of British Columbia, Vancouver, BC V6T 1 Z2 Canada and Department of Mathematics, Chinese University Of Hong Kong, Shatin, Hong Kong.

E-mail address: wei@math.cuhk.edu.hk 